\title{
Phase-field modeling of proppant-filled fractures in a poroelastic medium
}

\author{
Sanghyun Lee ${ }^{\mathrm{a}}$, Andro Mikelićc ${ }^{\mathrm{c}}$, Mary F. Wheeler ${ }^{\mathrm{a}, *}$, Thomas Wick \\ ${ }^{a}$ The Institute for Computational Engineering and Sciences, The University of Texas at Austin, Austin, Texas 78712, USA \\ ${ }^{b}$ RICAM, Austrian Academy of Sciences Altenberger Str. 694040 Linz, Austria \\ ${ }^{c}$ Université de Lyon, Lyon, F-69003, France; Université Lyon 1, Institut Camille Jordan, UMR 5208, Bât. Braconnier, 43, Bd du 11 novembre \\ 1918, 69622 Villeurbanne Cedex, France
}

\begin{abstract}
In this paper we present a phase field model for proppant-filled fractures in a poroelastic medium. The formulation of the coupled system involves four unknowns; displacements, phase field, pressure, and proppant concentration. The two-field displacement phase-field system is solved fully-coupled and accounts for crack irreversibility. This solution is than coupled to the pressure equation via a fixed-stress iteration. The pressure is obtained by using a diffraction equation where the phase-field variable serves as an indicator function that distinguishes between the fracture and the reservoir. The transport of the proppant in the fracture is modeled by using a power-law fluid system. The numerical discretization in space is based on Galerkin finite elements for displacements and phase-field, and an enriched Galerkin method is applied for the pressure equation in order to obtain local mass conservation. The concentration is solved with cell-centered finite elements. Nonlinear equations are treated with Newton's method. Our developments are substantiated with several numerical examples in two and three dimensions.
\end{abstract}

Keywords: Phase field fracture, Hydraulic fracturing, Proppant transport, Quasi-Newtonian flow model

\section{Introduction}

Murray Roth, a VP at Global Consulting, has called proppant the greatest oilfield innovation of the 21st century. A proppant is a solid material, typically sand, treated sand or man-made ceramic materials, designed to keep an induced hydraulic fracture open, during or following a fracturing treatment. The objective of a hydraulic fracturing treatment in the oil industry is to increase the flow area exposed to the formation and then to connect the flow area to the wellbore along a high permeability path. Proppant is added to a fracking fluid which may vary in composition depending on the type of fracturing used, and can be gel, foam or slickwater-based. Although hydraulic fracturing was first performed in 1947 (in Kansas, using sand from the Arkansas river), wide-spread experimentation did not occur until the Barnett Shale play in the 80s, and usage has exploded in the first decade of this century. Expansion was aided by a landmark study conducted by the EPA (environmental protection agency) in 2004 which found that hydraulic fracturing posed no threat to underground drinking water supplies. Shortly afterwards, hydraulic fracturing was exempted from the Safe Drinking Water Act by the Bush administration in the Energy Policy Act of 2005.

Hydraulic fracturing has become a key element of natural gas development worldwide, and countries such as the Unites States, Canada, India, England and China are actively pursuing implementation of this technology to tap into this new source of energy. However, like any advanced technology, it has also raised questions about its long-term impact on the environment. Thus debate and research will continue well into the future and the role of more advanced models will be required for new and upcoming regulations.

\footnotetext{
${ }^{*}$ Corresponding author

Email addresses: shlee@ices.utexas.edu (Sanghyun Lee), mikelic@univ-lyon1.fr (Andro Mikelić), mf w@ices.utexas.edu (Mary F. Wheeler), thomas.wick@ricam. oeaw.ac.at (Thomas Wick)
} 

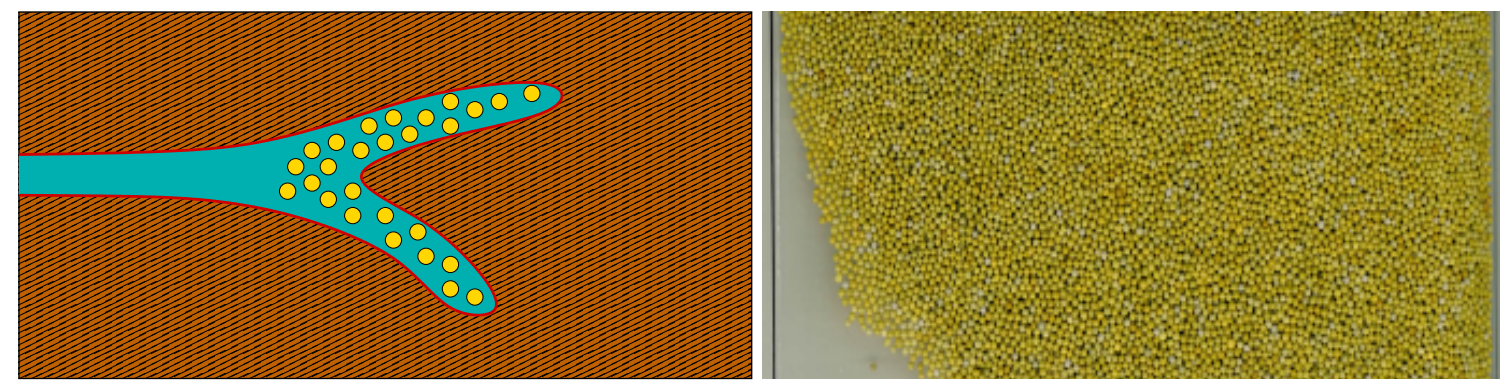

Figure 1: Illustration of proppant usage (yellow coated grains) preventing the fracture from closing (left). At right, real proppant grains used in hydraulic fracturing are shown.

The quality of proppant is designed to maintain lasting, high permeability under conditions of in situ stress and temperature. Since the goal in field operations is to distribute proppant optimally, accurate and efficient numerical models are essential in representing proppant transport in fractures. Here simulation is challenging because of the complex interactions between the fluid, particles and fracture walls. As described by [1] in the modeling of hydraulic fracturing, a variety of physical processes such as fluid flow, stress induced by fracture deformation, complex fluid rheology, and fracture propagation are occurring simultaneously. Slurry flow, gravitational settling, and proppant transport modeling for hydraulic fractures are currently poorly understood and modeling capability limited.

Mixing proppant with a Newtonian fluid has been investigated in [1, 28, 50, 68], and with polymers in [64], [24], [69], [48] to name a few. These numerical studies have been performed using boundary element methods, discrete fracture networks or extended/generalized finite elements and have mainly treated 2D planar fractures. A survey of different models for fracture propagation can be found in [79] and our focus in this paper will be on phase-field fracture models. Presently, the latter approach is subject of active research in both mathematical theory and applications. Based on variational principles, the phase-field technique provides an elegant way to approximate lower-dimensional surfaces and discontinuities.

Rewriting Griffith's model [38] for brittle fracture in terms of a variational formulation was first accomplished by Francfort and Marigo [33]. Later, in [54, 53], the authors refined modeling and material law assumptions to formulate an incremental thermodynamically consistent phase-field model for fracture propagation. Computational techniques, finite element analysis, and multiple examples and benchmarks from mechanical engineering have been proposed and studied in [14, 15, 54, 53, 16, 11, 12, 39, 65, 51, 3, 36]. Recent advances and numerical studies towards hydraulic fracturing and other multiphysics applications including thermo-elastic-plastic solids and coupling with a reservoir simulator have been considered in [61, 60, 77, 56, 55, 79, 52, 46]. Clearly, these cited works demonstrate that phase-field fracture modeling has tremendous potential tackling practical field problems.

More specifically, phase-field fracture modeling offers many advantages such as a fixed-grid topology that avoids expensive remeshing for resolving the exact fracture location. Thus, the model can be implemented easily to simulate both 2D and 3D crack propagation [12, 39, 80, 56, 55]. Therein, fracture nucleation, propagation, kinking, curvilinear path, branching, joining and handling large fracture networks are intrinsically determined. Moreover, the phasefield variable can be used to compute the crack opening displacement (e.g., [76]) as well as serving as an indicator function to formulate a pressure diffraction model in order to couple with phase-field fracture with other multiphysics phenomena [60]. On the other hand, the diffusive transition zone tends to smear out the sharp crack surface and the characteristic length-scale parameter $\varepsilon$ must be chosen accordingly, which is a particular modeling challenge in porous media [59]. For numerical treatment, an adaptive local grid refinement technique can be used to increase crack surface resolution while keeping the computational costs low [39]. Additionally, some of the challenges associated with a non-convex functional inherent in the phase field formulation have also been addressed in [13, 15, 54, 2, 39, 36].

Since phase-field modeling has attractive features as previously discussed, we further develop this methodology in the present paper in order to establish phase-field for quasi-Newtonian fluid and proppant transport combined with fracture propagation. Since correct proppant transport modeling is a major research topic as previously described, we consider a prototype transport equation that can later be extended to more complicated and realistic material parameter relationships such as slip velocity vectors, gravitational settling, and interaction of proppant with fracture walls. From an engineering point of view of view, an important ingredient for the transport equation is appropriate velocities that 
are obtained from the fracture pressure equation and which are locally mass conservative [72, 42]. An elegant way to satisfy local mass conservation in numerical simulations is based on an enriched Galerkin (EG) formulation [71] that we employ in this work.

In summary, the novelties of the present paper are:

- Derivation of a power-law lubrication equation for fracture flow;

- Spatial discretization of the pressure diffraction system with the enriched Galerkin (EG) method;

- Formulation of a fixed-stress algorithm for robust numerical treatment of a four-field system for coupling displacements, phase-field, pressure and proppant concentration.

The outline of this paper is as follows: In Section 2 we present notation, geometry and the basic equations. Next, in Section 3 numerical discretization is discussed. Then in Section 4, our solution algorithm is formulated. In Section 5. several numerical examples substantiate our modeling and numerical developments. The Appendix provides a detailed derivation of nonlinear Darcy's law for a power-law model for fracture flow.

\section{Modeling}

In order to fix ideas we start with the notation, geometry and a description of the Biot equations. The key idea is to split the original Biot system into flow and geomechanics using a fixed-stress scheme. For phase-field fracture modeling in porous media, the geomechanics equations are coupled with a phase-field equation including a crack irreversibility condition. The reservoir flow equation is combined with fracture flow by employing a pressure diffraction approach. In this work we extend fracture Newtonian flow to a quasi-Newtonian power-law model. An analysis of this system is provided in subsection 2.5. In the final subsection 2.7, a prototype model for proppant transport is suggested.

\subsection{Geometry and fracture surface description}

Let $C:=C(t)$ be a given open set diffeomorphic to an ellipsoid in $\mathbb{R}^{3}$ (a crack set), for every time $t \in[0, T]$. The fracture boundary is a closed surface $\partial C:=\partial C(t)$, changing in time and separating it from the surrounding poroelastic medium as illustrated in Figure 2

$$
\Lambda=\Omega \cup \bar{C}
$$

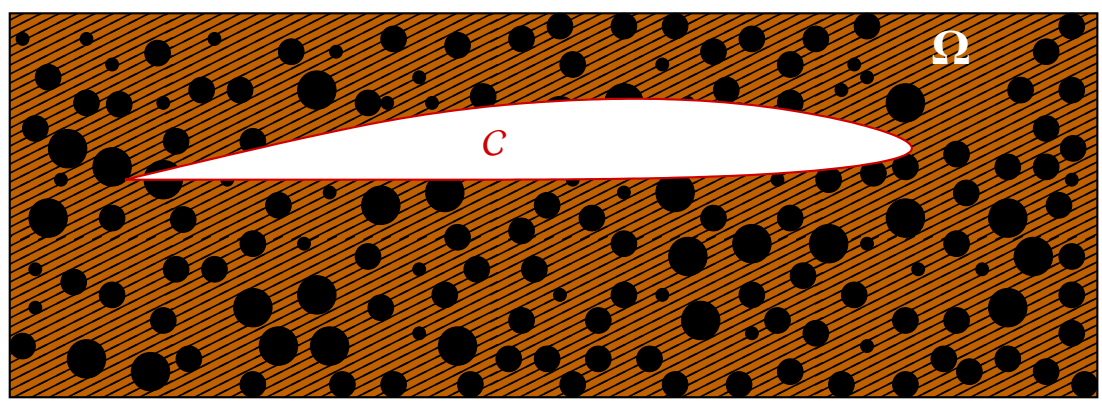

Figure 2: A crack $C$ is compactly contained in a porous medium $\Lambda:=\Omega \cup \bar{C}$. We assume the dimensions of the crack are much larger than the pore scale size (black dots) of the porous medium.

In most applications $C$ is a curved 3D domain, with one dimension significantly smaller than the other dominant two. Furthermore we suppose a flat middle surface of the fracture. An example is a penny shape crack as displayed in Figure 3. The surrounding poroelastic domain is $\Omega:=\Omega(t)=\Lambda \backslash \bar{C}$, where $\Lambda=(0, L)^{3} \supset \mathcal{C}$, i.e. the crack $C$ is contained compactly in $\Omega$ and does not reach the boundary. The boundary of $\Lambda=(0, L)^{3}$ is denoted by $\partial \Lambda$, which is divided into open 2D surfaces $\partial_{D} \Lambda$ and $\partial_{N} \Lambda$, with smooth boundaries. We assume that meas $\left(\partial_{D} \Lambda\right)>0$ and $\partial \Lambda=\overline{\partial_{D} \Lambda} \cup \overline{\partial_{N} \Lambda}$. Boundary conditions on $\partial_{D} \Lambda$ for the Biot system involve displacements and the normal fluxes and on $\partial_{N} \Omega$ traction as well as pressure. 


\subsection{Biot equations (without fractures)}

The quasi-static Biot equations in $\Omega \times(0, T]$ (see e.g. [74]) read as follows:

$$
\begin{gathered}
\sigma^{p o r}-\sigma_{0}=\mathcal{G} e(\mathbf{u})-\alpha p_{R} I ; \quad-\operatorname{div}\left(\sigma^{p o r}\right)=\rho_{b} \mathbf{g} ; \\
\partial_{t}\left(\frac{1}{M} p_{R}+\operatorname{div}(\alpha \mathbf{u})\right)+\operatorname{div}\left(\frac{K_{R}}{\eta_{R}}\left(\rho_{R} \mathbf{g}-\nabla p_{R}\right)\right)=\frac{q_{R}}{\rho_{R}},
\end{gathered}
$$

where $\sigma^{\text {por }}$ is the total poroelasticity tensor $(\mathrm{Pa}), \sigma_{0}$ is the reference state total stress, $\mathbf{u}: \Omega \times[0, T] \rightarrow \mathbb{R}^{3}$ is the solid's displacement (m), $p_{R}: \Omega \times[0, T] \rightarrow \mathbb{R}$ is the fluid reservoir pressure (Pa), and $q=q_{R} / \rho_{R}$ represents source terms. $\mathcal{G}$ is a constant symmetric positive definite rank-4 tensor. In most applications we model the stress $\mathcal{G} e(\mathbf{u})$ using a linear stress-strain relationship:

$$
\mathcal{G} e(\mathbf{u})=2 \mu e(\mathbf{u})+\lambda \operatorname{tr}(e(\mathbf{u})) I,
$$

where $e(\mathbf{u}):=\left(\nabla \mathbf{u}+\nabla^{T} \mathbf{u}\right) / 2$ is the linearized strain tensor, $I$ is the identity matrix, $\operatorname{tr}(\cdot)$ the trace operator, and $\mu$ and $\lambda$ are material parameters. Other parameters and unknowns are listed in Table 1

\begin{tabular}{|l|l|l|}
\hline \hline Symbol & Quantity & Unity \\
\hline$K_{R}$ & permeability of the poroelastic domain & Darcy \\
\hline$K_{F}$ & fracture permeability & Darcy \\
\hline$\rho_{b}$ & bulk density & $\mathrm{kg} / \mathrm{m}^{3}$ \\
\hline$\rho_{F}$ & reference state fracture fluid density & $\mathrm{kg} / \mathrm{m}^{3}$ \\
\hline$\rho_{R}$ & reference state pore fluid density & $\mathrm{kg} / \mathrm{m}^{3}$ \\
\hline$\eta_{F}$ & fracture fluid viscosity & $\mathrm{kg} / \mathrm{m} \mathrm{sec}$ \\
\hline$\eta_{R}$ & pore fluid viscosity & $\mathrm{kg} / \mathrm{m} \mathrm{sec}$ \\
\hline$\alpha$ & Biot's coefficient & $\mathrm{dimensionless}$ \\
\hline$M$ & Biot's modulus & $\mathrm{Pa}$ \\
\hline$c_{F}$ & fracture fluid compressibility & $1 / \mathrm{Pa}$ \\
\hline $\mathbf{g}$ & gravity & $\mathrm{m} / \mathrm{sec}^{2}$ \\
\hline
\end{tabular}

Table 1: Unknowns and effective coefficients with units.

\subsection{Specific remarks to fracture descriptions in porous media}

In classic fracture propagation references, e.g., [1, 34, 70], the crack $C$ is considered to be a lower dimensional manifold. This point of view is justified in solid mechanics. In case of a fluid-filled fracture, lubrication theory is applied to describe the fluid flow (see e.g. [1, 34, 45, 66]); namely one replaces moving boundary Stokes flow by a Reynolds equation.

However in our work fractures propagate in a poroelastic medium. Therefore, their description is obtained by upscaling the fluid-structure pore scale first principle equations. In this new setting the meaning of a Griffith's type surface energy is not clear and we choose to deal with a physical 3D fracture, to which we can attribute an energy term leading to propagation [59,61].

In the crack we consider incompressible viscous flow. Since this takes place in a thin domain, we replace the Navier-Stokes equations by the appropriate 3D Darcy law. Its derivation, and in particular the permeability calculation, is based on the lubrication approximation (see [73]). In the case of Newtonian incompressible viscous flow in the crack $C$, the 3D Darcy law using lower dimensional lubrication approximation is derived in [60]. The extension to quasi-Newtonian fracture flow is undertaken below.

\subsection{Power-law flow equations in the fracture}

We now turn to the description of the fracture pressure equation where we assume the fracturing fluid as quasiNewtonian. The motivation is that polymers change the fluid rheology; see [31] and references cited therein. We use the following density-pressure relationship

$$
\rho_{F}=\rho_{F}^{0} \exp \left(c_{F}\left(p_{F}-p_{F}^{0}\right)\right) \approx \rho_{F}^{0}\left[1+c_{F}\left(p_{F}-p_{F}^{0}\right)\right],
$$


where $\rho_{F}^{0}$ denotes the (constant) reference density, $p_{F}: \Omega \times[0, T] \rightarrow \mathbb{R}$ is the fluid fracture pressure (Pa), and $p_{F}^{0}$ is a given initial fluid fracture pressure. The fluid compressibility $c_{F}$ is assumed to be small and $\rho_{F} \approx \rho_{F}^{0}$. Nevertheless, even after linearization, compressibility effects are important in the pressure equation. Using the smallness of $c_{F}$, the fracture mass conservation equation is then given by:

$$
c_{F} \partial_{t} p_{F}+\operatorname{div}\left(\mathbf{v}_{F}\right)=\frac{q_{F}}{\rho_{F}^{0}} \quad \text { in } C \times(0, T],
$$

where $q_{F}$ is a source term and $\mathbf{v}_{F}$ is nonlinear Darcy's law defined below. To describe the quasi-Newtonian fluid, we introduce the following notation. Let the Frobenius matrix norm $|\cdot|$ be given by

$$
|\xi|^{2}=\operatorname{tr}\left(\xi \xi^{t}\right)=\xi: \xi=\sum_{i, j} \xi_{i j}^{2}, \xi \in \mathbb{R}^{9}
$$

The fracture viscosity $\eta_{F}^{e f f}$ is assumed to be a power of the second invariant $e_{I I}(\mathbf{v})=\operatorname{tr}\left(e(\mathbf{v})^{2}\right)$ of the rate of strain tensor $e(\mathbf{v})$ :

$$
\eta_{F}^{e f f}=\eta_{F} \dot{\gamma}^{m-1}=\eta_{F}|\sqrt{2} e(\mathbf{v})|^{m-1},
$$

where $\eta_{F}$ is the shear consistency of the proppant and $m>0$ is the power-law exponent, characterizing the strain rate sensitivity of the proppant; see [10] for more details. A prototype fracture model is a 3D penny-shape crack as shown in Figure 3 .

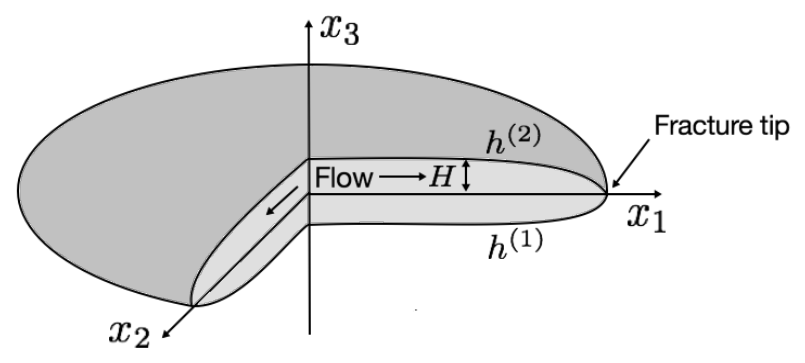

Figure 3: Sketch of the fracture region for a three-dimensional setting. The boundary $x_{3}=h^{(j)}\left(x_{1}, x_{2}, t\right), j=1,2$, moves in time

The fracture boundary is given by

$$
\begin{array}{lll}
x_{3}=h^{(2)}\left(x_{1}, x_{2}, t\right) & \text { for } x_{3} \geq 0, & t \in[0, T], \\
x_{3}=h^{(1)}\left(x_{1}, x_{2}, t\right) & \text { for } x_{3}<0, & t \in[0, T],
\end{array}
$$

and

$$
C(t)=\left\{\left(x_{1}, x_{2}, x_{3}\right) \mid h^{(1)}\left(x_{1}, x_{2}, t\right)<x_{3}<h^{(2)}\left(x_{1}, x_{2}, t\right),\left(x_{1}, x_{2}\right) \in \omega\right\}, t \in[0, T],
$$

where $\omega$ is a smooth bounded domain in $\mathbb{R}^{2}$. Note that $h^{(j)}, j=1,2$ describes a moving boundary.

The fracture $C$ is characterized by the ratio $\varepsilon$ between its characteristic width $H$ in the direction $x_{3}$ and its characteristic horizontal length $L$ in directions $x_{1}$ and $x_{2}$; a sketch is provided in Figure 3 . We assume that width $H$ of the fracture is much larger than the pore size of the porous medium and recall that a coupled model must be taken into account. Here $H:=c_{0} l^{2 / 3}$, where $l$ is the pore size. Due to the small ratio $\varepsilon=H / L$ the results from the Appendix apply and the relative velocity satisfies nonlinear Darcy's law

$$
\mathbf{v}_{F}=\frac{K_{F}}{\eta_{F}^{1 / m}}\left|\mathbf{f}-\nabla p_{F}\right|^{\frac{1}{m}-1}\left(\mathbf{f}-\nabla p_{F}\right)+\left(0,0, v_{L}\right),
$$

where $|x|=\sqrt{x_{1}^{2}+x_{2}^{2}+x_{3}^{2}}$ and $\mathbf{f}$ includes boundary conditions from the derivation and the gravity in $f_{3}$. The leak off term $v_{L}$ and the fracture absolute permeability $K_{F}$ are given by (102) and (101), respectively in the Appendix. In addition, $K_{F}$ depends on the shape of the crack and on $\eta_{F}$ and $m$. 
The functions $h^{(1)}$ and $h^{(2)}$ can be considered as negative and positive displacements of the crack, respectively. An illustration is provided in Figure 3 i.e., $h^{(1)}+h^{(2)}=2 \mathbf{u}_{\text {width }}$ where $\mathbf{u}_{\text {width }}$ denotes the half-width of the fracture. The characteristic width $H$ of the fracture is chosen as $H:=\max \left\{-h^{(1)}, h^{(2)}\right\}$. Note that for a penny shaped fracture $C$ the dominant components of the velocity are two dimensional.

Remark 1. The surfaces $h^{(2)}$ and $h^{(1)}$ meet at $x_{3}=0$, which is the tip region. The nonlinear filtration law (7) is valid everywhere except at the tip zone, where degeneration takes place. Thus, excluding the tip zone and avoiding singular behavior at the interface between the fracture and the reservoir arising from the permeability jump, we determine a new permeability-viscosity ratio at the interface by interpolation. We finally recall that our crack permeability law is based on the assumption that cracks curvature does not change too rapidly.

Modeling the moving interface $\partial C$ is challenging. To begin, we notice that the crack domain $C(t)$ penetrates the poroelastic medium $\Omega(t)$ with the interface velocity $v_{I}$. As discussed in [60], Sec 2.3., the interface-corrected continuity of fluxes (the first principle Rankine-Hugoniot conditions) lead to the following interface conditions

$$
\begin{gathered}
p_{R}=p_{F} \quad \text { and } \quad \sigma^{p o r} \mathbf{n}=-p_{F} \mathbf{n} \quad \text { on the moving interface } \partial C, \\
\mathbf{v}_{F} \cdot \mathbf{n}=\mathbf{v}_{R} \cdot \mathbf{n}=\frac{K_{R}}{\eta_{R}}\left(\rho_{R} \mathbf{g}-\nabla p_{R}\right) \cdot \mathbf{n} \quad \text { on the moving interface } \partial C,
\end{gathered}
$$

where $\mathbf{v}_{F}$ and $\mathbf{v}_{R}$ are fracture and reservoir velocities, respectively. Furthermore, $\mathbf{n}$ denotes the normal exterior to the fracture.

Condition (8) is a simplification of the pressure interface conditions and more careful modeling by upscaling indicates presence of an additional term; the interested reader is referred to [49].

\subsection{A pressure diffraction problem}

We have seen that under the assumption of the weak compressibility of the fluid, the mass conservation equation is given by $(2)$ in the poroelastic part $\Omega$ and by $(3)$ in the fracture $C$. The pressure diffraction problem that combines reservoir and fracture flow reads:

$$
\theta(\mathbf{x}, t) \partial_{t} p+\operatorname{div}\left(-K_{e f f}(\nabla p, \mathbf{x}, t)(\nabla p-\tilde{\mathbf{G}})\right)=\bar{q}-\chi_{C(t)} \frac{\partial v_{L}}{\partial x_{3}} \quad \text { in } \quad \Lambda,
$$

where

$$
\begin{gathered}
\theta(\mathbf{x}, t):=\chi_{\Omega(t)} \frac{1}{M}+\chi_{C(t)} c_{F}, \quad \bar{q}:=\bar{q}(\mathbf{x}, t):=\chi_{\Omega(t)} \frac{q_{R}}{\rho_{R}}-\chi_{\Omega(t)} \alpha \partial_{t}(\operatorname{div} \mathbf{u})+\chi_{C(t)} \frac{q_{F}}{\rho_{F}} \\
\tilde{\mathbf{G}}:=\chi_{\Omega(t)} \rho_{R} \mathbf{g e}_{3}+\chi_{C(t)} \mathbf{f}, \quad K_{e f f}(\nabla p, \mathbf{x}, t)=\chi_{\Omega(t)} \frac{K_{R}}{\eta_{R}}+\chi_{C(t)} \frac{K_{F}}{\eta_{F}^{1 / m}}\left|\mathbf{f}-\nabla p_{F}\right|^{1 / m-1}
\end{gathered}
$$

and the flow velocity is defined as

$$
\mathbf{v}:=-K_{e f f}(\nabla p, \mathbf{x}, t)(\nabla p-\tilde{\mathbf{G}}) .
$$

According to our assumption that we consider a flat fracture, $\frac{\partial v_{L}}{\partial x_{3}}$ only acts in the third dimension. More details are provided in the Appendix. Moreover, in the general case we would deal with a normal derivative with respect to middle surface.

The indicator functions are given by

$$
\chi_{\Omega(t)}:=1 \quad \text { in } \Omega(t), \quad \text { and } \quad \chi_{\Omega(t)}:=0 \text { in } C(t),
$$

such that $\chi_{C(t)}=1-\chi_{\Omega}$ following [60]. The formulation contains the time-dependent interface $\Gamma(t):=\bar{C}(t) \cap \bar{\Omega}(t)$ between $\Omega$ and $C$ as the crack propagates.

The interface conditions are given by

$$
\begin{aligned}
{[p] } & =0, \\
{\left[-K_{e f f}(\nabla p, \mathbf{x}, t)(\nabla p-\tilde{\mathbf{G}})\right] \cdot \mathbf{n} } & =v_{L} n_{3},
\end{aligned}
$$


where $[\cdot]$ denotes the jump. Here, $n_{3}$ is the third component of the unit normal vector to the interface.

We impose the following initial conditions

$$
\left.p\right|_{t=0}=p_{F}^{0} \quad \text { in } \bar{C}(0) \quad \text { and }\left.\quad p\right|_{t=0}=p_{R}^{0} \quad \text { in } \Omega(0) .
$$

For simplicity we suppose homogeneous Neumann conditions (no flow) on $\partial \Lambda$ :

$$
\left(-K_{e f f}(\nabla p, \mathbf{x}, t)(\nabla p-\tilde{\mathbf{G}})\right) \cdot \mathbf{n}=0 .
$$

In the following, we study existence and uniqueness for the diffraction problem $[10)-(16)$. Let us introduce the Banach space $W$ by

$$
W=\left\{p \in L^{a}\left(0, T ; W^{1, a}(\Lambda)\right) \mid \nabla p \in L^{2}(\Omega \times(0, T))^{3} \text { and } \nabla p \in L^{1+1 / m}(C \times(0, T))^{3}\right\},
$$

where $a=\min \{2,1+1 / m\}$. The space $W^{1, a}$ contains functions from $L^{a}$ with their gradients also in $L^{a}$. The weak (variational or virtual work) formulation for problem $(10)-(16)$ reads:

Problem 2 (Pressure diffraction problem on time-dependent domains). Find $p \in L^{\infty}\left(0, T ; L^{2}(\Lambda)\right), \partial_{t} p \in L^{2}(\Omega \times(0, T))$, $\nabla p \in L^{\infty}\left(0, T ; L^{a}(\Lambda)\right)^{3}, \nabla p \in L^{\infty}\left(0, T ; L^{2}(\Omega)\right)^{3}$ and $\nabla p \in L^{\infty}\left(0, T ; L^{1+1 / m}(C)\right)^{3}$ such that

$$
\begin{aligned}
& \int_{0}^{T} \int_{\Lambda} \theta(\mathbf{x}, t) \partial_{t} p \psi d x d t+\int_{0}^{T} \int_{\Lambda} K_{e f f}(\nabla p, \mathbf{x}, t)(\nabla p-\tilde{\mathbf{G}}) \nabla \psi d x d t \\
&= \int_{0}^{T} \int_{\Lambda} \bar{q} \psi d x d t+\int_{0}^{T} \int_{C(t)} \frac{\partial \psi}{\partial x_{3}} v_{L} d x d t \quad \forall \psi \in W, \\
&\left.p\right|_{t=0}=p^{0}=\chi_{\Omega(0)} p_{R}^{0}+\chi_{C(0)} p_{F}^{0} .
\end{aligned}
$$

Problems of type $[18]-(19)$ have been treated in [44], page 232, for the case $\tilde{\mathbf{G}}=0$ and $m=1$. In [60] the case $m=1$ has been considered and in the present work we extend these results to a quasilinear parabolic PDE corresponding to $m \neq 1$.

In order to overcome the challenge of moving domains, we use a standard approach arising from moving boundary problems (see e.g., [32]) and transform the current configuration at time $t$ back to its initial configuration at time $t=0$. This transformation can be realized by using the corresponding displacement field $\mathbf{u}$ in $\Omega(t)$. Let $y \in \Omega(0)$. We make the following assumptions on the current displacement, the deformation gradient and its determinant:

$$
\begin{aligned}
& x=\mathbf{u}(y, t)+y, \quad t \in[0, T], \\
& \mathbf{u} \in C^{1}([0, T] \times \bar{\Lambda})^{3}, \quad \frac{D y}{D x} \in W^{1, \infty}((0, T) \times \Lambda)^{9}, \quad J(t)=\operatorname{det} \frac{D y}{D x}>0 .
\end{aligned}
$$

Then, the interface $\Gamma(t)$ is $C^{1}$. Moreover, the normal vector $\mathbf{n}$ and the velocity $\mathbf{v}=\partial_{t} \mathbf{u}$ are continuous. We have the following result

Proposition 3. We assume that $K_{R} \in L^{\infty}(\Lambda)^{9}$ is a uniformly elliptic matrix. For the data we assume $p^{0} \in W^{1, \infty}(\Lambda)$, $\bar{q} \in L^{\infty}\left((0, T ; C(\bar{\Lambda}))^{3}\right), v_{L} \in C^{1}\left([0, T] ; L^{\infty}(\Lambda)\right)$, and $\left.\tilde{\mathbf{f}} \in W^{1, \infty}\left((0, T ; C(\bar{\Lambda}))^{3}\right)\right)$. With the further assumptions in (21), the problem (18)-(19) has a unique solution $p \in L^{\infty}\left(0, T ; L^{2}(\Lambda)\right), \partial_{t} p \in L^{2}((0, T) \times \Lambda), \nabla p \in L^{\infty}\left(0, T ; L^{a}(\Lambda)\right)^{3}$, $\nabla p \in L^{\infty}\left(0, T ; L^{1+1 / m}(C)\right)^{3}$, and $\nabla p \in L^{\infty}\left(0, T ; L^{2}(\Omega)\right)^{3}$.

Proof. We work with the diffeomorphism $x=\mathbf{u}(y, t)+y$ and introduce the space $W^{0}$, which contains the functions of the space $W$ mapped to the initial domain; for details of the definition of such a space we refer the reader to [32].

The inverse diffeomorphism transforms $\Gamma(t)$ to $\Gamma(0)$ and the part of $\Omega$ far from the interface is unchanged (similar to the arbitrary Lagrangian-Eulerian approach in fluid-structure interaction [27, 41]). The weak formulation (18) transforms to

$$
\begin{gathered}
\int_{0}^{T} \int_{\Lambda} \theta^{0} \partial_{t} \tilde{p} \tilde{\psi} J(t) d y d t+\int_{0}^{T} \int_{\Lambda} K_{e f f}^{0}\left(\frac{D y}{D x} \nabla_{y} \tilde{p}-\tilde{\mathbf{G}}^{0}\right) \frac{D y}{D x} \nabla_{y} \tilde{\psi} J(t) d y d t \\
=\int_{0}^{T} \int_{\Lambda} \bar{q}^{0} \tilde{\psi} J(t) d y d t+\int_{0}^{T} \int_{C(0)} \frac{D y}{D x} \nabla \psi \mathbf{e}_{3} v_{L}^{0} J(t) d x d t, \quad \psi \in W,
\end{gathered}
$$


and where $\tilde{\psi} \in W^{0}$. Here $\tilde{p}(\mathbf{y}, t)=p(\mathbf{x}(\mathbf{y}, t), t), \tilde{\mathbf{f}}(\mathbf{y}, t)=\mathbf{f}(\mathbf{x}(\mathbf{y}, t), t)$ and $\theta^{0}:=\chi_{\Omega(0)} \frac{1}{M}+\chi_{C(0)} c_{F}$. Respectively,

$$
\begin{aligned}
& \bar{q}^{0}:=\bar{q}(\mathbf{y}, t):=\chi_{\Omega(0)} \frac{\tilde{q}_{R}(\mathbf{y}, t)}{\rho_{R}}-\chi_{\Omega(0)} \alpha \partial_{t}(\operatorname{div} \mathbf{u}(y, t))+\chi_{C(0)} \frac{\tilde{q}_{F}(\mathbf{y}, t)}{\rho_{F}} \\
& \tilde{\mathbf{G}}^{0}:=\chi_{\Omega(0)} \rho_{R} \mathbf{g e}_{3}+\chi_{C(0)} \tilde{\mathbf{f}}(y, t) \\
& K_{e f f}^{0}:=K_{e f f}^{0}\left(\nabla_{y} \tilde{p}, \mathbf{y}, t\right)=\chi_{\Omega(0)} \frac{K_{R}(\mathbf{x}(\mathbf{y}, t))}{\eta_{R}}+\chi_{C(0)} \frac{K_{F}}{\eta_{F}^{1 / m}}\left|\tilde{\mathbf{f}}-\frac{D y}{D x} \nabla_{y} \tilde{p}_{F}\right|^{1 / m-1} .
\end{aligned}
$$

Letting $\tilde{\psi}=\partial_{t} \tilde{p}$, we have

$$
\begin{gathered}
\int_{0}^{T} \int_{\Lambda} \theta^{0}\left(\partial_{t} \tilde{p}\right)^{2} J(t) d y d t+\int_{0}^{T} \int_{\Lambda} K_{e f f}^{0}\left(\frac{D y}{D x} \nabla_{y} \tilde{p}-\tilde{\mathbf{G}}^{0}\right) \cdot \frac{D y}{D x} \partial_{t} \nabla_{y} \tilde{p} J(t) d y d t \\
=\int_{0}^{T} \int_{\Lambda} \bar{q}^{0} J(t) \partial_{t} \tilde{p} d y d t+\int_{0}^{T} \int_{C(0)} \frac{D y}{D x} \frac{\partial}{\partial x_{3}} \partial_{t} \tilde{p} \mathbf{e}_{3} v_{L} J(t) d y d t .
\end{gathered}
$$

We transform the second term on the left hand side of 23 . Let us introduce the abbreviation

$$
\Pi=\frac{D y}{D x} \nabla_{y} \tilde{p}-\tilde{\mathbf{G}}^{0}, \quad \Pi(0)=\nabla p^{0}-\left.\tilde{\mathbf{G}}^{0}\right|_{t=0}
$$

Then we have

$$
\begin{aligned}
& \int_{\Lambda} K_{e f f}^{0} \Pi \cdot \frac{D y}{D x} \partial_{t} \nabla_{y} \tilde{p} J(t) d y=\underbrace{\int_{\Lambda} K_{e f f}^{0} \Pi \cdot \partial_{t} \Pi J(t) d y}_{=I_{1}} \\
& +\underbrace{\int_{\Lambda} K_{e f f}^{0} \Pi \cdot \partial_{t} \tilde{\mathbf{G}}^{0} J(t) d y-\int_{\Lambda} K_{e f f}^{0} \Pi \cdot\left(\partial_{t}\left(\frac{D y}{D x}\right) \frac{D y}{D x} \Pi\right) J(t) d y-\int_{\Lambda} K_{e f f}^{0} \Pi \cdot\left(\partial_{t}\left(\frac{D y}{D x}\right) \frac{D y}{D x}^{-1} \tilde{\mathbf{G}}\right) J(t) d y}_{=I_{2}} \\
& =I_{1}+I_{2} .
\end{aligned}
$$

We note that 21 and $\left.\mathbf{f} \in W^{1, \infty}\left((0, T ; C(\bar{\Lambda}))^{3}\right)\right)$ imply $\left.\tilde{\mathbf{G}}^{0} \in W^{1, \infty}\left((0, T ; C(\bar{\Lambda}))^{3}\right)\right)$. It is analogous for $K_{F}$ and $K_{e f f}^{0}$. Using the assumed regularity of the coefficients yields

$$
\left|I_{2}\right| \leq C\left(\int_{\Omega(0)}|\Pi|^{2} J(t) d y+\int_{C(0)}|\Pi|^{1+1 / m} J(t) d y+1\right)
$$

Next we transform the term $I_{1}$ :

$$
\begin{gathered}
I_{1}=\partial_{t}\left(\int_{\Omega(0)} \frac{1}{2 \eta_{R}} K_{R} \Pi \cdot \Pi J d y+\int_{C(0)} \frac{m}{(m+1) \eta_{F}^{1 / m}} K_{F}^{0} \Pi \cdot \Pi|\Pi|^{1 / m-1} J d y\right) \\
-\int_{\Omega(0)} \frac{1}{2 \eta_{R}} K_{R} \Pi \cdot \Pi \partial_{t} J d y-\int_{C(0)} \frac{m}{(m+1) \eta_{F}^{1 / m}} K_{F}^{0} \Pi \cdot \Pi|\Pi|^{1 / m-1} \partial_{t} J d y-\int_{C(0)} \frac{\partial_{t} K_{F}^{0}}{\eta_{F}^{1 / m}}|\Pi|^{1 / m+1} J d y .
\end{gathered}
$$

Inserting (24)-(26) into (23) and using the hypothesis on the coefficients, we obtain

$$
\begin{aligned}
& \int_{0}^{t} \int_{\Lambda}\left(\partial_{\tau} \tilde{p}\right)^{2} J(t) d y d \tau+\int_{\Omega(0)}|\Pi|^{2}(t) J(t) d y+\int_{C(0)}|\Pi|^{1+1 / m}(t) J(t) d y \\
& \leq C\left(\int_{\Omega(0)}|\Pi|^{2}(0) d y+\int_{C(0)}|\Pi|^{1+1 / m}(0) d y+\int_{0}^{t} \int_{C(t)}\left|v_{L}\right|^{m+1} d x d t+\int_{0}^{t} \int_{\Lambda}\left(q^{0}\right)^{2} J d y d \tau\right. \\
&\left.\quad+\int_{0}^{t}\left(\int_{\Omega(0)}|\Pi|^{2}(\tau) J(\tau) d x+\int_{C(0)}|\Pi|^{1+1 / m}(\tau) J(\tau) d y\right) d \tau+1\right)
\end{aligned}
$$


Gronwall's inequality implies the a priori estimate

$$
\|\nabla p\|_{L^{\infty}\left(0, T ; L^{2}(\Omega(t))\right)}+\|\nabla p\|_{L^{\infty}\left(0, T ; L^{1+1 / m}(C(t))\right)}+\left\|\partial_{t} p\right\|_{L^{2}(\Omega \times(0, T))} \leq C .
$$

Proving existence and uniqueness for problem $(18)-(19)$ is based on a Galerkin technique and thus straightforward. A sketch of the proof is provided in the following:

1. We introduce finite dimensional spaces $W_{1} \subset W_{2} \subset \cdots \subset W_{N} \subset W$ and construct a finite dimensional approximation $p_{N}$.

2. By the estimate (27), $p_{N}$ exists on $(0, T)$ and satisfies estimate $(28)$, which is independent of $N$.

3. We then let $N \rightarrow \infty$. By estimate [28) and elementary weak and weak-* compactness, there exists a subsequence $\left\{p_{l}\right\}_{l \in \mathbb{N}}$, such that

$$
\begin{aligned}
p_{l} \rightarrow p & \text { strongly in } L^{2}\left(0, T ; L^{a}(\Lambda)\right), \\
\partial_{t} p_{l} \rightarrow \partial_{t} p & \text { weakly in } L^{2}(\Lambda \times(0, T)), \\
p_{l} \rightarrow p & \text { weakly in } W \\
\nabla p_{l} \rightarrow \nabla p & \text { weak-* in } L^{\infty}\left(0, T ; L^{a}(\Lambda)\right) .
\end{aligned}
$$

4. Passing to the limit is now straightforward, except in the quasilinear term involving the nonlinear viscosity law. The latter one is monotone in $\nabla p$ and we apply Minty's lemma and write the Galerkin approximation of the problem (18)-(19) in the equivalent form

$$
\begin{gathered}
\int_{0}^{T} \int_{\Lambda} \theta \partial_{t} p_{N}\left(\psi-p_{N}\right) d x d t+\int_{0}^{T} \int_{\Lambda} K_{e f f}(p, \mathbf{x}, t)(\nabla \psi)(\nabla \psi-\tilde{\mathbf{G}}) \nabla\left(\psi-p_{N}\right) d x d t \\
-\int_{0}^{T} \int_{\Lambda} \bar{q}\left(\psi-p_{N}\right) d x d t-\int_{0}^{T} \int_{C(t)} \frac{\partial\left(\psi-p_{N}\right)}{\partial x_{3}} v_{L} d x d t \geq 0, \quad \forall \psi \in W_{M} ; M \leq N .
\end{gathered}
$$

Using that

$$
\lim _{N \rightarrow \infty} \int_{0}^{T} \int_{\Lambda}\left(\chi_{\Omega} \frac{K_{R}}{\eta_{R}}+\chi_{C} \frac{K_{F}}{\eta_{F}^{1 / m}}|\mathbf{f}-\nabla \psi|^{1 / m-1}\right) \nabla\left(\psi-p_{N}\right) d x d t=\int_{0}^{T} \int_{\Lambda}\left(\chi_{\Omega} \frac{K_{R}}{\eta_{R}}+\chi_{C} \frac{K_{F}}{\eta_{F}^{1 / m}}|\mathbf{f}-\nabla \psi|^{1 / m-1}\right) \nabla(\psi-p) d x d t
$$

we conclude that $p$ satisfies variational inequality 33 for every $\psi \in W$. After applying once more Minty's lemma we conclude that $p$ is a solution for problem (18)-(19).

Uniqueness is a consequence of the regularity and monotonicity.

Remark 4. We emphasize that the previous theoretical considerations only hold for smooth cracks without branching and joining. Nevertheless, the associated computational method is still applicable to such settings, which is demonstrated in Section 5

Remark 5 (Lower-dimensional fracture pressure equations). Many research publications concentrate on the accurate description of the fluid equation in the fracture. Often, the fracture is identified as a lower dimensional manifold (a line in 2D or a plane in 3D) on which the lubrication equation is used, for instance in [34. 45. 66]. Our strategy is different because the phase-field requires a volume approximation and can be used as indicator function.

\subsection{The displacement phase-field system}

To compute the fractured displacement field, we follow the approach by Miehe et al. [54], which is based on Francfort and Marigo [33]. Here, the fracture length (or surface area) can be approximated using an elliptic functional [4, 5] as, thereby introducing a variable $\varphi$, referred to as the phase-field variable hereafter. The phase field variable $\varphi$ is defined as a continuous quantity that smoothly varies between 0 and 1 on $\Lambda$ for $0 \leq t \leq T$. This smooth interpolation introduces a diffusive zone of width $\varepsilon$, where $\varepsilon$ is a regularization parameter with the fracture $C$ being approximated 
as a three-dimensional domain. As is usually done in fracture settings in variational methods, we assume crack irreversibility. This condition is an inequality constraint in time and reads:

$$
\partial_{t} \varphi \leq 0
$$

For the complete quasi-static variational formulation with the irreversibility constraint, we refer to [61]. In this article we consider only the incremental formulation. The corresponding incremental form is given by

$$
\varphi \leq \varphi^{\text {old }}
$$

where $\varphi^{\text {old }}$ denotes later the previous time step solution.

The function spaces are chosen as

$$
V_{\mathbf{u}}:=H_{0}^{1}(\Lambda), \quad W_{\varphi}:=H^{1}(\Lambda), \quad \text { and } \quad W_{\varphi^{o l d}}^{\text {in }}:=\left\{w \in H^{1}(\Lambda) \mid w \leq \varphi^{\text {old }} \leq 1 \text { a.e. on } \Lambda\right\} .
$$

Furthermore, for modeling of fractures with pressure terms (see [59, 61]), we define $\varphi_{+}:=\max \{0, \varphi\}$. The incremental formulation that describes quasi-static fracture propagation in poro-elasticity has been derived in [61] and reads:

Formulation 6. Let the pressure $p$ be given. Find vector-valued displacements $\mathbf{u} \in V_{\mathbf{u}}$ and a scalar-valued phase-field variable $\varphi \in W_{\varphi^{\text {old }}}^{\text {in }}$ such that

$$
\begin{aligned}
& \int_{\Lambda}\left((1-\kappa) \varphi_{+}^{2}+\kappa\right)(\mathcal{G} e(\mathbf{u}): e(\mathbf{w})) d x-\int_{\Lambda}(\alpha-1)\left(\varphi_{+}^{2} p, \operatorname{div} \mathbf{w}\right) d x+\int_{\Lambda}\left(\varphi_{+}^{2} \nabla p \cdot \mathbf{w}\right) d x=0, \forall \mathbf{w} \in V_{\mathbf{u}}, \\
& \int_{\Lambda}(1-\kappa)\left(\varphi_{+} G e(\mathbf{u}): e(\mathbf{u})(\psi-\varphi)\right) d x-\int_{\Lambda} 2(\alpha-1)\left(\varphi_{+} p(\operatorname{div} \mathbf{u})(\psi-\varphi)\right) d x \\
& +\int_{\Lambda} 2\left(\varphi_{+}(\nabla p \cdot \mathbf{u})(\psi-\varphi)\right) d x+\int_{\Lambda} G_{c}\left(-\frac{1}{\varepsilon}(1-\varphi)(\psi-\varphi)+\varepsilon(\nabla \varphi \cdot(\nabla(\psi-\varphi)))\right) d x \geq 0, \forall \psi \in W_{\varphi} .
\end{aligned}
$$

Furthermore, $\kappa>0$ and $\varepsilon>0$ are the phase-field regularization parameters with $\kappa \ll \varepsilon[14]$ with $\kappa=10^{-10} h$. Here, $G_{c}$ stands for the critical energy release rate that is related to the fracture toughness.

Remark 7. The solution of the previous system depends on the choice of $\kappa$ and $\varepsilon$. As discussed in [14] and [11], $\kappa$ can be chosen very small at the machine tolerance level. The choice of $\varepsilon$ is restricted by the constraint $\varepsilon>h$. Several numerical simulations performed in [39] 78] and [46] for 2D and $3 D$ investigate the influence of $\varepsilon$.

\subsection{Proppant transport in the fracture $C$}

For modeling the proppant transport we solve an advective equation that determines the volumetric distribution of concentration $c$, see e.g., [1, 30].

Formulation 8. Find the concentration $c$ such that for all times $t \in(0, T]$ :

$$
\partial_{t}\left(\varphi^{\star} c\right)+\operatorname{div}\left(\mathbf{v}_{p} c\right)=Q c_{q} \frac{q_{F}}{\rho_{F}}, \quad \text { in } \Lambda(t) \times(0, T]
$$

where $\mathbf{v}_{p}=Q \mathbf{v}, Q>0$ is a coefficient for the velocity, and $\mathbf{v}$ is obtained from 11 . The initial condition is given by

$$
c(\mathbf{x}, 0)=c_{0}(\mathbf{x}), \quad \forall \mathbf{x} \in \Lambda,
$$

where $c$ is the species concentration advected, $c_{q}$ is a general source or sink term. As boundary conditions, we prescribe

$$
\mathbf{v}_{p} \cdot \mathbf{n}=0 \quad \text { on } \partial \Lambda \times(0, T]
$$


Various types of proppant velocity models (e.g. $\left.\mathbf{v}_{p}=Q \mathbf{v}-(1-c) \mathbf{v}_{s}\right)$ are discussed by [20, 22, 21, 63, 37] regarding the slip velocity vector $\left(\mathbf{v}_{s}\right)$ with settling of proppant. Those models are based on theoretical and experimental results with careful assumptions for proppant particle size, density, fracture width and fluid velocity, see [1, 8, 19, 75]. Recently, modeling of the proppant velocity parameter $Q$ is studied in [29]. The choice of a model for $\mathbf{v}_{p}$ is non-trivial since each has different assumptions with varying results. Therefore, in this work, we focus on a prototype model to observe the distribution of proppant in the fracture. We assume the proppant velocity is equal to the fluid velocity (no slip and settling) and instead of providing any specific $Q$, we restrict $Q$ to be a constant. Several numerical examples using prototype models with different choices for $Q$ are presented in Section 5. Complete studies with validations can be achieved once more experimental data are available.

\section{Numerical discretization}

In the previous section, we derived a phase-field model with four equations for the unknowns: displacements, phase-field, pressure, and proppant concentration. In this section, we formulate temporal, spatial discretization and linearization. The displacement phase-field system is treated with Galerkin finite elements, the pressure diffraction problem is solved with an enriched Galerkin (EG) method in order that the flow is locally conservative. The transport equation is treated with cell-centered finite elements. Temporal discretization is performed with A-stable backward Euler finite difference scheme.

We consider a shape regular mesh family $\left\{\mathcal{T}_{h}\right\}_{h>0}$, and we assume that each mesh $\mathcal{T}_{h}$ is a subdivision of $\bar{\Lambda}$ made of disjoint elements $T$, i.e., squares when $d=2$ or cubes when $d=3$. Each subdivision is assumed to exactly approximate the computational domain, thus $\bar{\Lambda}=\cup_{T \in \mathcal{T}_{h}} T$. The diameter of an element $K \in \mathcal{T}_{h}$ is denoted by $h$ and we denote $h_{\min }$ for the minimum. For any integer $k \geq 1$ and $T \in \mathcal{T}_{h}$, we denote by $\mathbb{Q}^{k}(T)$ the space of scalar-valued multivariate polynomials over $T$ of partial degree of at most $k$. The vector-valued counterpart of $\mathbb{Q}^{k}(T)$ is denoted $\mathbb{Q}^{k}(T)$. In addition, we define a partition of the time interval $0=: t^{0}<t^{1}<\cdots<t^{N}:=T$ and denote the time step size by $\delta t:=t^{n}-t^{n-1}$.

\subsection{Numerical discretization for flow/pressure using enriched Galerkin (EG)}

In porous media flow it is well known that the velocities should be locally mass conservative [42, 72]. This can either be achieved by post-processing of the velocities or by a direct solution in which the function space is enriched. We employ the latter method, the enriched Galerkin (EG), that was first proposed in [71] and is extended in [47]. Throughout the remaining sections, we assume the gravity $\tilde{\mathbf{G}} \equiv 0$ and that fluid leakage is zero.

\subsubsection{Approximation in space and time}

The EG space approximation $P$ of the pressure function $p(\mathbf{x}, t)$ is approximated by piecewise polynomials in the following finite element space:

$$
V_{h}^{E G}:=M_{0}^{k}\left(\mathcal{T}_{h}\right)+M^{0}\left(\mathcal{T}_{h}\right)
$$

We define,

$$
M^{k}\left(\mathcal{T}_{h}\right):=\left\{p \in L^{2}(\Lambda) \mid p_{\left.\right|_{T}} \in \mathbb{Q}_{k}(T), \forall T \in \mathcal{T}_{h}\right\}
$$

and denote $M_{0}^{k}\left(\mathcal{T}_{h}\right)$ by

$$
M_{0}^{k}\left(\mathcal{T}_{h}\right):=M^{k}\left(\mathcal{T}_{h}\right) \cap \mathbb{C}(\Lambda),
$$

the subspace of $M^{k}\left(\mathcal{T}_{h}\right)$ consisting of the globally continuous piecewise continuous functions $(\mathbb{C})$.

We let $\mathcal{E}_{h}$ be the set of all edges/faces and $\mathcal{E}_{h}^{o}$ and $\mathcal{E}_{h}^{\partial}$ are the collection of all interior and boundary edges/faces, respectively. In the following, we use the notation for $2 \mathrm{D}$ edges but the results hold analogously for $3 \mathrm{D}$ faces. The boundary edges $\mathcal{E}_{h}^{\partial}$ can be further decomposed into $\mathcal{E}_{h}^{\partial}=\mathcal{E}_{h}^{D, \partial} \cup \mathcal{E}_{h}^{N, \partial}$, where $\mathcal{E}_{h}^{D, \partial}$ is the collection of edges where Dirichlet boundary conditions are imposed, while $\mathcal{E}_{h}^{N, \partial}$ is the collection of edges where Neumann boundary conditions are imposed. In addition, we denote that $\mathcal{E}_{h}^{1}:=\mathcal{E}_{h}^{o} \cup \mathcal{E}_{h}^{D, \partial}$ and $\mathcal{E}_{h}^{2}:=\mathcal{E}_{h}^{o} \cup \mathcal{E}_{h}^{N, \partial}$. For any $e \in \mathcal{E}_{h}^{o}$, let $T^{+}$and $T^{-}$be two neighboring elements such that $e=\partial T^{+} \cap \partial T^{-}$. We denote by $h_{e}$ the maximum length of the edges $e$. Let $\mathbf{n}^{+}$and $\mathbf{n}^{-}$ be the outward normal unit vectors to $\partial T^{+}$and $\partial T^{-}$, respectively $\left(\mathbf{n}^{ \pm}:=\mathbf{n}_{\mid T^{ \pm}}\right)$. For any given function $\xi$ defined on the triangulation $\mathcal{T}_{h}$, we denote $\xi^{ \pm}$by the restrictions of $\xi$ to $T^{ \pm}$. 
The space $H^{k}\left(\mathcal{T}_{h}\right)(k \in \mathbb{R})$ is the set of element-wise $H^{k}$ functions on $\mathcal{T}_{h}$, and $L^{2}\left(\mathcal{E}_{h}\right)$ refers to the set of functions whose traces on the elements of $\mathcal{E}_{h}$ are square integrable.

In addition, we define the average $\{\cdot\}$ as follows:

$$
\{w\}:=\frac{1}{2}\left(w^{+}+w^{-}\right) \text {for } w \in L^{2}\left(\mathcal{T}_{h}\right), \quad \text { and } \quad\{w\}:=w \text { for } e \in \mathcal{E}_{h}^{\partial}
$$

The jump across the interior edge will defined as

$$
\llbracket w \rrbracket=w^{+} \mathbf{n}^{+}+w^{-} \mathbf{n}^{-} \text {on } e \in \mathcal{E}_{h}^{o}, \quad \text { and } \quad \llbracket w \rrbracket=w \mathbf{n} \text { for } e \in \mathcal{E}_{h}^{\partial} .
$$

Next, we define indicator functions to determine the domains for the fracture and the reservoir. As discussed in (12), once the phase field value $\varphi\left(t^{n+1}\right)$ is computed, we define,

$$
\chi_{C}^{n+1}:=\frac{1-H_{h}^{\star}\left(\varphi\left(t^{n+1}\right)\right)}{2}, \quad \chi_{\Omega}^{n+1}:=\frac{1+H_{h}^{\star}\left(\varphi\left(t^{n+1}\right)\right)}{2}
$$

where $H_{h}^{\star}($.$) is an approximation of the Heaviside function given as$

$$
H_{h}^{\star}(s)=\left\{\begin{array}{cl}
1 & \text { if } s>0.5+D_{\epsilon}, \\
-1 & \text { if } s<0.5-D_{\epsilon}, \\
\frac{s-0.5}{D_{\epsilon}} & \text { otherwise. }
\end{array}\right.
$$

Here we set $D_{\epsilon}=0.1$.

Let the approximation of $P\left(\mathbf{x}, t^{n}\right), 0 \leq n \leq N$ be denoted by $P^{n}$. Then, the discretized problem reads as follows:

Formulation 9. Let $\mathbf{u}\left(t^{n+1}\right), \varphi\left(t^{n+1}\right)$, and the previous time step pressure solution $P^{n}$ be given;

$$
\text { Find } P^{n+1} \in V_{h}^{E G} \text { such that } \mathcal{S}\left(P^{n+1}, w\right)=0, \quad \forall w \in V_{h}^{E G},
$$

where $\mathcal{S}$ is the IIPG method [23] and the variational form defined as

$$
\begin{aligned}
\mathcal{S}\left(P^{n+1}, w\right):=\sum_{T \in \mathcal{T}_{h}} \int_{T} \theta^{n+1} & \frac{P^{n+1}-P^{n}}{\delta t} w d x+\sum_{T \in \mathcal{T}_{h}} \int_{T} K_{e f f}^{n+1} \nabla P^{n+1} \cdot \nabla w d x \\
& \quad-\sum_{e \in \mathcal{E}_{h}^{1}} \int_{e}\left\{K_{e f f}^{n+1} \nabla P^{n+1}\right\} \llbracket w \rrbracket d \gamma+\sum_{e \in \mathcal{E}_{h}^{1}} \int_{e} \frac{1}{h_{e}} K_{e f f_{\mid e}}^{n+1} \llbracket P^{n+1} \rrbracket \llbracket w \rrbracket d \gamma+\sum_{T \in \mathcal{T}_{h}} \int_{T} \bar{q}^{n+1} w d x
\end{aligned}
$$

Here,

$$
\begin{aligned}
\theta^{n+1} & :=\chi_{\Omega}^{n+1} \frac{1}{M}+\chi_{C}^{n+1} c_{F}, \quad \bar{q}^{n+1}:=\chi_{\Omega}^{n+1}\left(\frac{q_{R}^{n+1}}{\rho_{R}^{n+1}}-\alpha \partial_{t}\left(\operatorname{div} \mathbf{u}\left(t^{n+1}\right)\right)\right)+\chi_{C}^{n+1} \frac{q_{F}^{n+1}}{\rho_{F}^{n+1}}, \\
K_{e f f}^{n+1} & :=\chi_{\Omega}^{n+1} \frac{K_{R}}{\eta_{R}}+\chi_{C}^{n+1} \frac{K_{F}}{\eta_{F}^{1 / m}}\left|\mathbf{f}-\nabla P^{n+1}\right|^{1 / m-1} .
\end{aligned}
$$

Remark 10. The spatial and temporal discretization of the displacements is introduced in the next section. For this reason we still deal with $\partial_{t}\left(\operatorname{div} \mathbf{u}\left(t^{n+1}\right)\right)$ in the above system. 


\subsubsection{Computational treatment of the fracture permeability $K_{F}$}

Following the Appendix, the fracture permeability can be further approximated as:

$$
K_{F}=\left[\begin{array}{ccc}
K^{H O M} & 0 & 0 \\
0 & K^{H O M} & 0 \\
0 & 0 & K^{H O M}
\end{array}\right] .
$$

In our numerical simulations, we use the diagonal form of $K_{F}$ in (43) rather than (101). This is justified because $H^{1+r^{*}}=H^{2+1 / m}$ and $K^{H O M}$ is of order $H^{r^{*}}=H^{1+1 / m}$, resulting in the off-diagonal terms being much smaller than the diagonals. From the Appendix, we know that

$$
K^{H O M}(x, t)=-\frac{r-1}{r}\left(\left|x_{3}-\frac{h^{(1)}+h^{(2)}}{2}\right|^{r /(r-1)}-\left|\frac{h^{(1)}-h^{(2)}}{2}\right|^{r /(r-1)}\right) .
$$

We notice since $h^{(1)} \leq 0$ and $h^{(2)} \geq 0$ (see Figure 3 that $K^{\text {HOM }}$ is always nonnegative.

Remark 11 (Further possible simplifications). If we further assume $x_{3}=0$ and symmetry of the crack opening displacement $h^{1}=-h^{2}$, we obtain

$$
K^{H O M}(x, t)=-\frac{r-1}{r}\left(-\left|\frac{2 h^{(1)}}{2}\right|^{r /(r-1)}\right)=\frac{r-1}{r}\left(\left|h^{(1)}\right|^{r /(r-1)}\right)
$$

In the Newtonian case, $r=2$, we obtain the expression from [60], formula (2.7):

$$
K^{H O M}(x, t)=\frac{1}{2}\left(h^{(1)}\right)^{2} .
$$

\subsection{Numerical discretization of displacements and phase-field}

For spatial discretization of the displacement-phase-field system, we define a fully-coupled Euler-Lagrange formulation for treating $\mathbf{U}$ and $\Phi$ (approximating $\mathbf{u}, \phi$ ) simultaneously. We consider a time-discretized system in which time enters through the crack irreversibility condition. The latter constraint leads to a variational inequality system. The spatial discretized solution variables are $\mathbf{U} \in \mathbb{C}^{1}\left([0, T] ; \mathbb{V}_{0}\left(\mathcal{T}_{h}\right)\right)$ and $\Phi \in \mathbb{C}^{1}\left([0, T] ; \mathbb{Z}\left(\mathcal{T}_{h}\right)\right)$, where

$$
\begin{aligned}
& \mathbb{V}_{0}\left(\mathcal{T}_{h}\right):=\left\{W \in \mathbb{C}^{0}\left(\bar{\Lambda} ; \mathbb{R}^{d}\right) \mid W=\mathbf{0} \text { on } \partial \Lambda,\left.W\right|_{K} \in \mathbb{Q}^{1}(K), \forall K \in \mathcal{T}_{h}\right\}, \\
& \mathbb{Z}\left(\mathcal{T}_{h}\right):=\left\{Z \in \mathbb{C}^{0}(\bar{\Lambda} ; \mathbb{R})\left|Z^{n+1} \leq Z^{n} \leq 1, Z\right|_{K} \in \mathbb{Q}^{1}(K), \forall K \in \mathcal{T}_{h}\right\}
\end{aligned}
$$

In the following, we formulate a standard variational system of Formulation 6 and we denote by $\mathbf{U}^{n}, \Phi^{n}$ the time approximation of $\mathbf{U}\left(t^{n}\right), \Phi\left(t^{n}\right)$ respectively. Formulating a monolithic system, we extrapolate $\Phi$ (denoted by $E(\Phi)$ ) in order to avoid an indefinite Hessian matrix:

$$
E\left(\Phi^{n}\right)=\Phi^{n-2}+\frac{\left(t-t^{n-1}-t^{n-2}\right)}{\left(t-t^{n-1}\right)-\left(t-t^{n-1}-t^{n-2}\right)}\left(\Phi^{n-1}-\Phi^{n-2}\right) .
$$

This heuristic procedure has been shown to be an efficient and robust method, see [39]. We then obtain the following system:

Formulation 12. Given the initial conditions $\mathbf{U}^{0}:=\mathbf{U}(0)$ and $\Phi^{0}:=\Phi(0)$ and $P^{n+1}$, we seek $\left\{\mathbf{U}^{n+1}, \Phi^{n+1}\right\} \in \mathbb{V}_{0}\left(\mathcal{T}_{h}\right) \times$ $\mathbb{Z}\left(\mathcal{T}_{h}\right)$ such that

$$
A\left(\mathbf{U}^{n+1}, \Phi^{n+1}\right)\left(\mathbf{w}, \psi-\Phi^{n+1}\right) \geq 0 \quad \forall\{\mathbf{w}, \psi\} \in \mathbb{V}_{0}\left(\mathcal{T}_{h}\right) \times \mathbb{Z}\left(\mathcal{T}_{h}\right),
$$


where the semilinear form is given by

$$
\begin{aligned}
A\left(\mathbf{U}^{n+1}, \Phi^{n+1}\right)\left(\mathbf{w}, \psi-\Phi^{n+1}\right) & \\
\quad & \int_{\Lambda}(1-k)\left(E\left(\Phi^{n+1}\right)^{2}+k\right) \sigma^{+}\left(\mathbf{U}^{n+1}\right): e(\mathbf{w}) d \mathbf{x}+\int_{\Lambda} \sigma^{-}\left(\mathbf{U}^{n+1}\right): e(\mathbf{w}) d \mathbf{x} \\
& -\int_{\Lambda}(\alpha-1) E\left(\Phi^{n+1}\right)^{2} P^{n+1} \operatorname{div}(\mathbf{w}) d \mathbf{x}+\int_{\Lambda} E\left(\Phi^{n+1}\right)^{2} \nabla P^{n+1} \cdot \mathbf{w} d \mathbf{x} \\
& +(1-k) \int_{\Lambda} \Phi^{n+1} \sigma^{+}\left(\mathbf{U}^{n+1}\right): e\left(\mathbf{U}^{n+1}\right) \cdot\left(\psi-\Phi^{n+1}\right) d \mathbf{x}-2(\alpha-1) \int_{\Lambda} \Phi^{n+1} P^{n+1} \operatorname{div}\left(\mathbf{U}^{n+1}\right) \cdot\left(\psi-\Phi^{n+1}\right) d \mathbf{x} \\
& +\int_{\Lambda} 2 \Phi^{n+1} \nabla P^{n+1} \cdot \mathbf{U}^{n+1} \cdot\left(\psi-\Phi^{n+1}\right) d \mathbf{x} \\
& -G_{c} \int_{\Lambda} \frac{1}{\varepsilon}\left(1-\Phi^{n+1}\right) \cdot\left(\psi-\Phi^{n+1}\right) d \mathbf{x}+G_{c} \int_{\Lambda} \varepsilon \nabla \Phi^{n+1} \cdot \nabla\left(\psi-\Phi^{n+1}\right) d \mathbf{x},
\end{aligned}
$$

The solution of this system is discussed in Section 4.3 .

We decompose the stress tensor (i.e., the energy) into tensile $\sigma^{+}$and compressive $\sigma^{-}$parts for modeling of shear forces under compression as in [54,6].

Remark 13 (Further remarks on time-dependencies). The full system is time-dependent although not all equations contain time derivatives. The pressure and concentration equations have a time derivative whereas 'time' in the phase field equation enters through the irreversibility constraint. The displacement solution changes in time since the time-dependent variables of the other two equations enter.

For completeness, we define the directional derivative $A^{\prime}\left(\mathbf{U}^{n+1}, \Phi^{n+1}\right)\left(\delta \mathbf{U}^{n+1}, \delta \Phi^{n+1}, \mathbf{w}, \psi\right)$, which is required for Newton's method. We notice that several terms in the $\varphi$-variable vanish due to a time-lagged extrapolation. We seek $\left\{\delta \mathbf{U}^{n+1}, \delta \Phi^{n+1}\right\} \in \mathbb{V}_{0}\left(\mathcal{T}_{h}\right) \times \mathbb{Z}\left(\mathcal{T}_{h}\right)$ such that

$$
\begin{aligned}
& A^{\prime}\left(\mathbf{U}^{n+1}, \Phi^{n+1}\right)\left(\delta \mathbf{U}^{n+1}, \delta \Phi^{n+1}, \mathbf{w}, \psi-\Phi^{n+1}\right) \\
& =\int_{\Lambda}\left((1-k) E\left(\Phi^{n+1}\right)^{2}+k\right) \sigma^{+}\left(\delta \mathbf{U}^{n+1}\right): e(\mathbf{w}) d \mathbf{x}+\int_{\Lambda} \sigma^{-}\left(\delta \mathbf{U}^{n+1}\right): e(\mathbf{w}) d \mathbf{x} \\
& \quad+(1-k) \int_{\Lambda} \delta \Phi^{n+1} \sigma^{+}\left(\mathbf{U}^{n+1}\right): e\left(\mathbf{U}^{n+1}\right) \cdot\left(\psi-\Phi^{n+1}\right) d \mathbf{x}+(1-k) \int_{\Lambda} 2 \Phi^{n+1} \sigma^{+}\left(\delta \mathbf{U}^{n+1}\right): e\left(\mathbf{U}^{n+1}\right) \cdot\left(\psi-\Phi^{n+1}\right) d \mathbf{x} \\
& \quad-2(\alpha-1) P^{n+1} \int_{\Lambda}\left(\delta \Phi^{n+1} \operatorname{div}\left(\mathbf{U}^{n+1}\right)+\Phi^{n+1} \operatorname{div}\left(\delta \mathbf{U}^{n+1}\right) \cdot\left(\psi-\Phi^{n+1}\right)\right) d \mathbf{x} \\
& \quad+2 \int_{\Lambda} \delta \Phi^{n+1} \nabla P^{n+1} \cdot \mathbf{U}^{n+1} \cdot\left(\psi-\Phi^{n+1}\right) d \mathbf{x}+2 \int_{\Lambda} \Phi^{n+1} \nabla P^{n+1} \cdot \delta \mathbf{U}^{n+1} \cdot\left(\psi-\Phi^{n+1}\right) d \mathbf{x} \\
& \quad+G_{c} \int_{\Lambda} \frac{1}{\varepsilon} \delta \Phi^{n+1} \cdot\left(\psi-\Phi^{n+1}\right) d \mathbf{x}+G_{c} \int_{\Lambda} \varepsilon \nabla \delta \Phi^{n+1} \cdot \nabla \psi d \mathbf{x} \geq 0
\end{aligned}
$$

for all $\{\mathbf{w}, \psi\} \in \mathbb{V}_{0}\left(\mathcal{T}_{h}\right) \times \mathbb{Z}\left(\mathcal{T}_{h}\right)$.

\subsection{Numerical discretization of proppant transport}

In this subsection, we concentrate on the numerical discretization of proppant transport described in Section 2.7 . The space approximation $C$ of the concentration function $c(\mathbf{x}, t)$ is approximated using piecewise constant given in the finite element space,

$$
\mathbb{M}\left(\mathcal{T}_{h}\right):=\left\{W \in \mathbb{C}^{0}(\bar{\Lambda} ; \mathbb{R})|W|_{T} \in \mathbb{Q}^{0}(T), \forall T \in \mathcal{T}_{h}\right\}
$$

We denote the approximation of $C\left(\mathbf{x}, t^{n}\right)$ by $C^{n}$. 
Formulation 14. Assume $\mathbf{U}^{n}, \Phi^{n}$, and $P^{n+1}$ are given values at time $t^{n}$ with $C(\mathbf{x}, 0)=C^{0}$, where $C^{0}$ is an approximation of the initial condition $c\left(t^{0}\right)$. Let $C^{n}$ is given, we seek $C^{n+1} \in \mathbb{C}^{0}\left([0, T] ; \mathbb{M}\left(\mathcal{T}_{h}\right)\right)$ so that

$$
\begin{aligned}
\sum_{T \in \mathcal{T}_{h}} \int_{T} \frac{C^{n+1}-C^{n}}{\delta t} w d x- & \sum_{T \in \mathcal{T}_{h}} \int_{T} Q C^{n+1} \mathbf{V}^{n+1} \cdot \nabla w d x-\sum_{T \in \mathcal{T}_{h}} \int_{T} \frac{q_{F}^{-}\left(t^{n+1}\right)}{\rho_{F}^{n+1}} c_{q}\left(t^{n+1}\right) C^{n+1} w d x \\
& +\sum_{e \in \mathcal{E}_{h}^{o}} \int_{e} Q C^{*} \mathbf{V}^{n+1} \cdot \mathbf{n} \llbracket w \rrbracket d \gamma-\sum_{T \in \mathcal{T}_{h}} \int_{T} Q \frac{q_{F}^{+}\left(t^{n+1}\right)}{\rho_{F}^{n+1}} c_{q}\left(t^{n+1}\right) w d x=0, \quad \forall \omega \in \mathbb{M}\left(\mathcal{T}_{h}\right)
\end{aligned}
$$

Here the source terms are $0<c_{q} \leq 1, q_{F}^{+}:=\max \left(0, q_{F}\right), q_{F}^{-}:=\min \left(0, q_{F}\right)$ and upwind value $C^{*}$ is defined as

$$
C_{\mid e}^{*}:= \begin{cases}C^{-} & \text {if } \mathbf{V} \cdot \mathbf{n} \geq 0 \\ C^{+} & \text {if } \mathbf{V} \cdot \mathbf{n}<0 .\end{cases}
$$

Such conservative flux variables $\mathbf{V}^{n+1}$ can be obtained as follows. Let $P^{n+1}$ be the solution to the (41), we define the flux variables $\mathbf{V}^{n+1}$ at time step $t^{n+1}$, by the following procedure:

$$
\begin{aligned}
& \left.\mathbf{V}^{n+1}\right|_{T}=-K_{e f f}^{n+1} \nabla P^{n+1}, \quad \forall T \in \mathcal{T}_{h}
\end{aligned}
$$

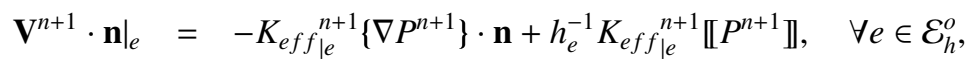

where $\mathbf{n}$ is the unit normal vector of the boundary edge $e$ of $T$, in which the value of the normal component of $\mathbf{V}^{n}$. The detailed proof of local and global conservation properties of such defined flux variables as well as their approximation estimate is given in [47].

\section{Coupling algorithms and numerical solution of nonlinear and linear systems}

In this section, we propose coupling algorithms to compute the unknowns $p, \mathbf{u}, \varphi$, and $c$ that are obtained by solving Formulation 9,12 and 14 , respectively.

\subsection{The global algorithm and mesh adaptivity}

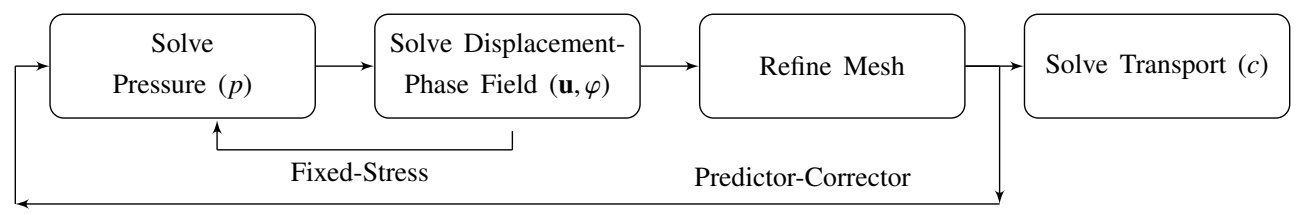

Figure 4: Global algorithm flowchart for each time step including the mesh refinement step.

The global algorithm is illustrated in Figure 4. Here we combine monolithic and splitting algorithms which is augmented with local mesh adaptivity. First, we solve for the degenerated pressure diffraction problem. Then, the displacement-phase-field is solved by an efficient and robust monolithic algorithm [39, 46]. The coupling of the latter problem to the pressure diffraction is realized with fixed-stress splitting [24, 58, 67]. The motivation in applying an iterative coupling algorithm is that a monolithic approach to the multiphysics problem is challenging due to the nonlinear coupling terms and the requirement for different discretization schemes. Moreover, future extensions to multiphase flow add more complexities. In addition, fixed stress splitting allows for straightforward coupling of fracture mechanics modules to reservoir simulators for production studies. After having obtained $p, \mathbf{u}$ and $\varphi$ we use these computed values to solve for the proppant transport. Then we proceed to the next time step and start from the beginning again.

For local mesh adaptivity, a fixed value for $\varepsilon$ is prescribed a priori that should be maintained throughout the entire computation. However, we must ensure that $\varepsilon>h$ holds true in the crack region. For this purpose we employ a recently proposed predictor-corrector mesh refinement technique [39]. Since the refinement indicator therein is 
based on the value of the phase-field variable, adaptivity can be performed after solving the phase-field system as shown in Figure 4. The phase-field equation is linked through fixed-stress to the pressure equation. The corrector step is performed after solving these systems and before solving the proppant transport. When the crack grows and the adaptive scheme detects that a step must be re-done, the entire fixed-stress step (corrector) needs to be solved again depending upon how many mesh levels are enforced. The additional numerical solution has the same cost as the predictor step and is therefore an expensive operation. However, in [39] (2D) and [46] (3D) it has been demonstrated that this procedure is efficient and accurate in comparison to global and a priori local mesh refinement. Indeed, the big advantage is that the mesh is only refined with respect to the unknown crack location and works for curvilinear cracks, branching and joining in two and three spatial dimensions.

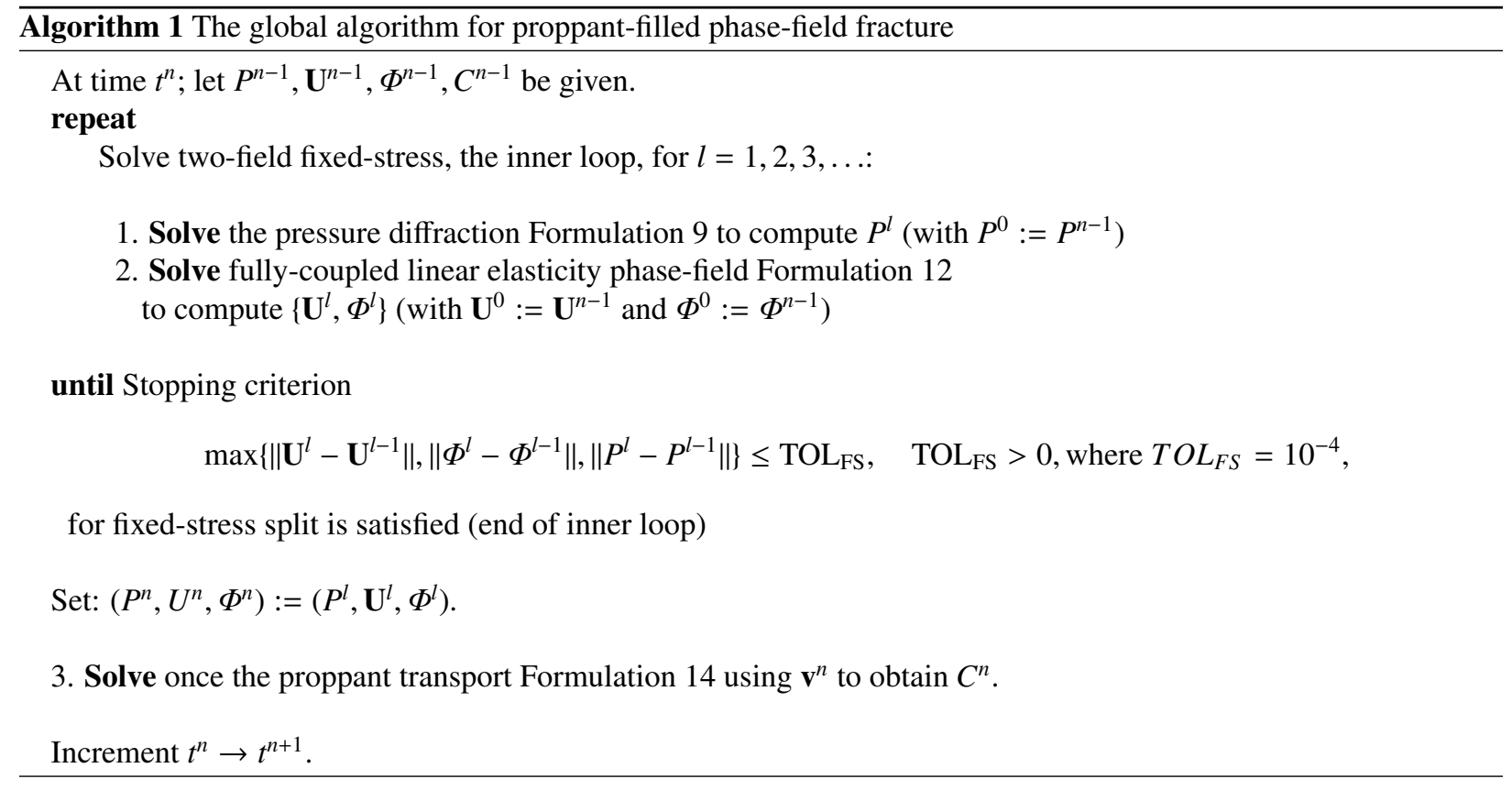

\subsection{Fixed-stress iteration between the displacement-phase-field system and pressure diffraction}

The fixed-stress iteration decouples pressure and geomechanics. And our principle idea follows the standard literature [24, 58, 67]. Extending fixed-stress to phase-field fractures, we also need to include the phase-field equation into the fixed-stress iteration. One approach is to have two loops: an outer fixed-stress iteration and an inner iteration between displacements and phase-field [60]. Another idea is to keep the fixed-stress iteration but to replace the inner iteration by a quasi-monolithic solve for the displacement-phase-field system [46]. Therefore, we restrict our presentation in this paper to the algorithm presented next.

Applying Algorithm 2 to the concrete equations yields: Given $P^{n}, \mathbf{U}^{n}$ and $\Phi^{n}$ setting the initial fixed-stress values $P^{0}:=P^{n}, \mathbf{U}^{0}:=\mathbf{U}^{n}$ and $\Phi^{0}:=\Phi^{n}$ and solve for $l=1,2,3, \ldots$ :

$$
\text { Given }\left\{\mathbf{U}^{l-1}, \Phi^{l-1}\right\} \text {, find } P^{l} \text { such that } \mathcal{S}\left(P^{l}, w ; \mathbf{U}^{l-1}, \Phi^{l-1}\right)=0, \quad \forall w \in V_{h}^{E G} \text {. }
$$

In the second step of fixed-stress, we solve:

$$
\text { Given } P^{l} \text {, find }\left\{\mathbf{U}^{l}, \Phi^{l}\right\} \text { such that } A\left(\mathbf{U}^{l}, \Phi^{n} ; P^{l}\right)\left(\mathbf{w}, \psi-\Phi^{l}\right) \geq 0, \quad \forall\{\mathbf{w}, \psi\} \in \mathbb{V}_{0}\left(\mathcal{T}_{h}\right) \times \mathbb{Z}_{\left(\mathcal{T}_{h}\right)}
$$

The numerical solution of each subproblem is briefly outlined in Section 4.3. Here, we stress that efficient and robust solution methods for the single subproblems have been developed in other papers and we refer to them.

\subsection{Nonlinear and linear solvers}

Algorithm 2 presents the fixed-stress phase field approach for fluid filled fractures in which the geomechanicsphase-field system is coupled to the pressure diffraction problem. Here, the pressure diffraction Formulation 9 is 


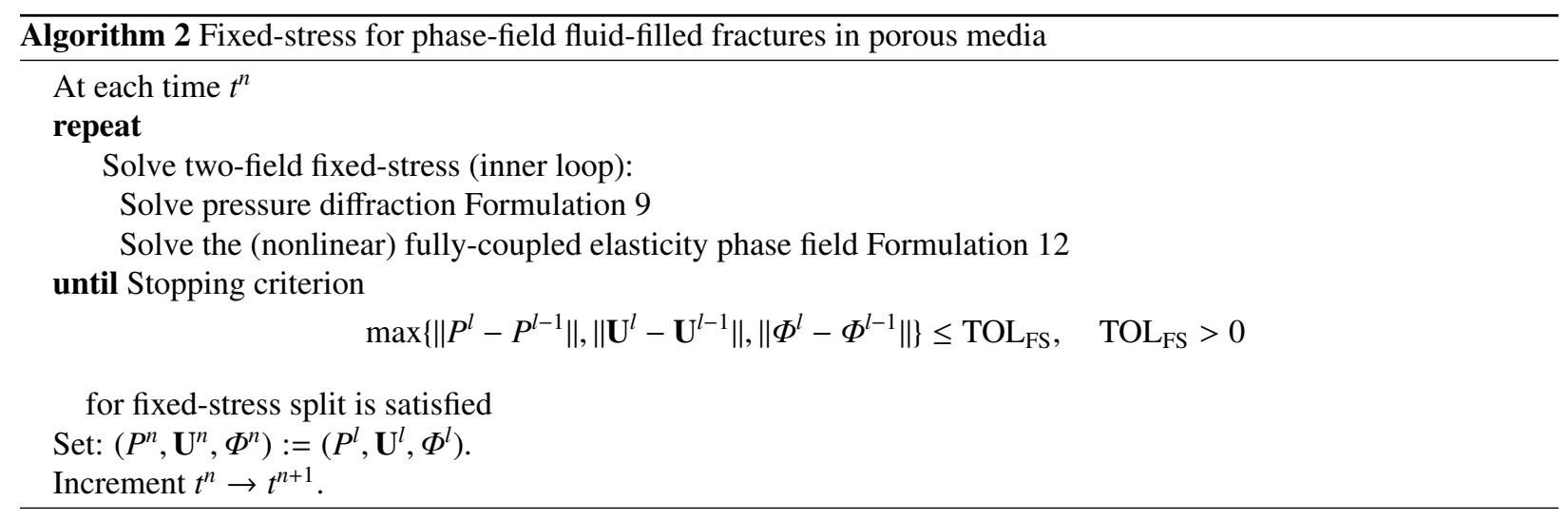

nonlinear for $m \neq 1$ due to the power-law fluid in the fracture; in the Newtonian case for $m=1$ the pressure problem is linear. In both cases a Newton solver with backtracking line search is employed and which converges for $m=1$ in one single iteration. Within Newton's method, the Jacobian is analytically derived in order to have optimal convergence properties. The nonlinear variational inequality presented in Formulation 12 is solved with a second Newton method in which two nonlinear iterations are combined. The first Newton iteration is required to solve the nonlinear forward problem $A\left(\mathbf{U}^{n+1}, \Phi^{n+1}\right)(\mathbf{w}, \psi)=0$ and the second (semi-smooth) Newton method is a realization of a primal-dual active set strategy to treat the crack irreversibility $\Phi^{n+1} \leq \Phi^{n}$ constraint. The resulting scheme has been formulated in [39]. In order to enhance the convergence radius, a backtracking line search algorithm is employed. Within Newton's method the linear equations are solved with the generalized minimal residual method (GMRES) using diagonal blockpreconditioning from Trilinos [40]. Further details and performance of these schemes are presented in [39, 46, 80]. Finally, the transport Formulation 14 is linear and solved with GMRES.

\section{Numerical Examples}

We present in this section several examples with increasing complexity. In order to calibrate our code we first compare Newtonian fracture flow with our quasi-Newtonian model. In the examples thereafter, the proppant transport equation is included as well. The current programming code is an extension of [80, 46] based on the MPI parallel phase-field fracture framework [39] using deal.II [7] and p4est [17]. Despite the fact that our theory has been performed for three-dimensional problems, we also present 2D numerical examples in order to substantiate our developments.

\subsection{Comparing crack propagation using Newtonian versus power-law fluid models (without proppant)}

The motivation for this first test is to compare Newtonian fracture flow with our quasi-Newtonian model. In the domain $\Lambda=(0 \mathrm{~m}, 4 \mathrm{~m})^{2}$, we set a initial fracture centered at $(2 \mathrm{~m}, 2 \mathrm{~m})$ with radius $r=0.4 \mathrm{~m}$ with thickness of $2 h_{\min }$ as shown in the Figure 10 (a). The mechanical parameters are $G_{c}=1.0 \mathrm{~N} / \mathrm{m}, v=0.2$ and $E=10^{8} \mathrm{~Pa}$ in the homogeneous domain. The fluid is injected at the center of the fracture with the constant volume rate of $q_{F}=200 \mathrm{~m}^{3} / \mathrm{s}$ for point source injection in the first 40 time steps and then slightly reduced by $q_{F}=200 \mathrm{~m}^{3} / \mathrm{s} \times(100-n) / 60$ (where $n$ is the time step number) and $q_{L}=0$. The fluid parameters are given as $\eta_{F}=\eta_{R}=10^{-3} \mathrm{Ns} / \mathrm{m}^{2}, \rho_{R}=\rho_{F}=1000 \mathrm{~kg} / \mathrm{m}^{3}$. The diagonal entry of the permeability $K_{R}=\operatorname{diag}\left(k_{R}\right)$ is defined with its entry $k_{R}=10^{-12}$, and all other parameters are $c_{F}=10^{-10}$ and the Biot modulus is $M=2.5 \times 10^{8} \mathrm{~Pa}$. Here $\varepsilon=2 h_{\min }, h_{\min }=0.022 \mathrm{~m}$ and the time step is $\delta t=0.01 s$, with $T=3 s$. For the power law index, we use $m=1$ and $m=0.5$. As functional values we observe the length of the fracture and the highest pressure value. In our numerical results, we do not observe significant differences in the fracture length; see Figures 5. In Figure 6 we demonstrate predictor-corrector mesh refinement, where the mesh grows with the fracture. The pressure curves are observed in Figure 8 in which the Newtonian case shows a slightly higher pressure evolution. The corresponding pressure distribution is finally illustrated in Figure 7 

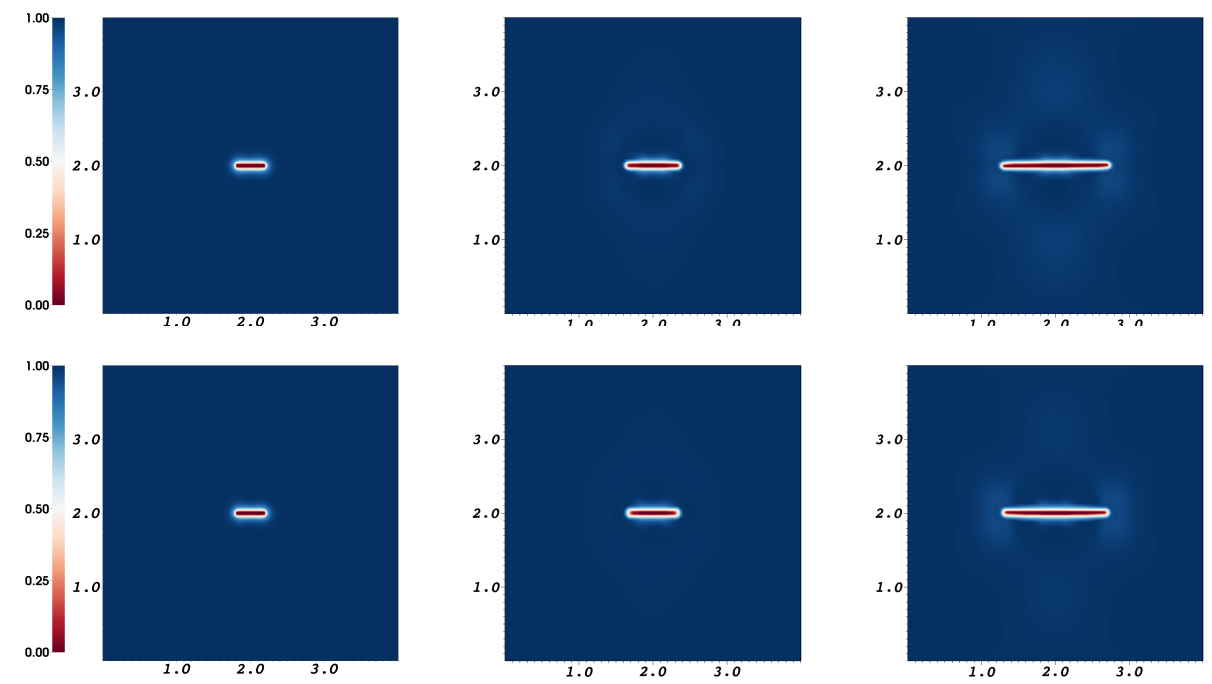

Figure 5: Example 5.1: Phase field values representing the fracture at times $T=0,0.3,0.5 \mathrm{~s}$ with $m=1$ (Newtonian fracture flow) on top and $m=0.5$ at the bottom. $\operatorname{Red}(\varphi=0)$ indicates the fracture.
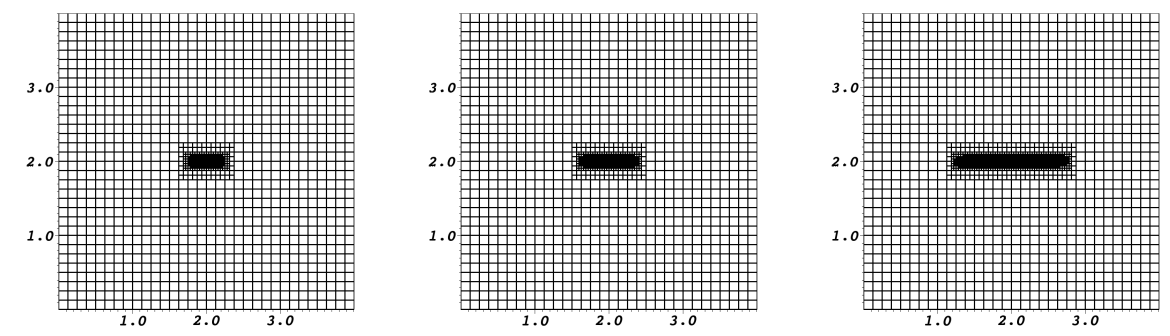

Figure 6: Example 5.1: Fracture at times $T=0,0.3,0.5 \mathrm{~s}$ with the Predictor-corrector mesh refinement where the mesh follows the fracture path.

\subsection{Tracer of the flow from injection to the production with a fixed fracture}

In the domain $\Lambda=(0 \mathrm{~m}, 10 \mathrm{~m})^{2}$, we measure the concentration values near the production source for two different cases; case i) high permeability (the diagonal entry of the permeability $K_{R}=\operatorname{diag}\left(K_{R}\right)=K_{F}=\operatorname{diag}\left(k_{F}\right)$ is defined with its entry $k_{R}=k_{F}=10^{-3}$ ) for the entire domain $\Lambda$ (see Figure 9 (a)) and case ii) low permeability $\left(k_{R}=10^{-12}\right.$ ) domain with a fixed fracture $\left(k_{F}=10^{-3}\right)$ in the middle of the domain as shown in the Figure 9 (e). The injection and production source are given at the point s $(5 \mathrm{~m}, 5 \mathrm{~m})$ and $(1 \mathrm{~m}, 1 \mathrm{~m})$, respectively. The injection and production rates are given as $q^{+}=100 \mathrm{~m}^{3} / \mathrm{s}$ and $q^{-}=-100 \mathrm{~m}^{3} / \mathrm{s}$ with the fluid parameters $\rho_{F}=\rho_{R}=1000 \mathrm{~kg} / \mathrm{m}^{3}, \eta_{F}=\eta_{R}=10^{-3} \mathrm{Ns} / \mathrm{m}^{2}$. Here, we choose $c_{q}=1$ for all following examples.

At each time $t=10$ and $t=1000$, figure 9(b)-(c) illustrates the concentration value for case i) and (f)-(g) for case ii). Finally, in Figure 9 (d) and (h), we plot the concentration values over the line $(1,1)-(2,2)$ as indicated by the arrows in (c)-(g). We observe that the fluid moves faster to the production source in case ii) when the same amount of the fluid is injected for both cases. This is because the fluid spreads to all direction for case i), and the flow is more localized and higher velocity occurs towards the production source for case ii).

\subsection{A single fracture with concentration in $2 D$ and $3 D$}

\subsection{1. $2 D$ setting}

In this section, we modify the proppant speed parameter $Q$ in (34) and observe distributions of proppant concentration depending on the fracture propagation. 

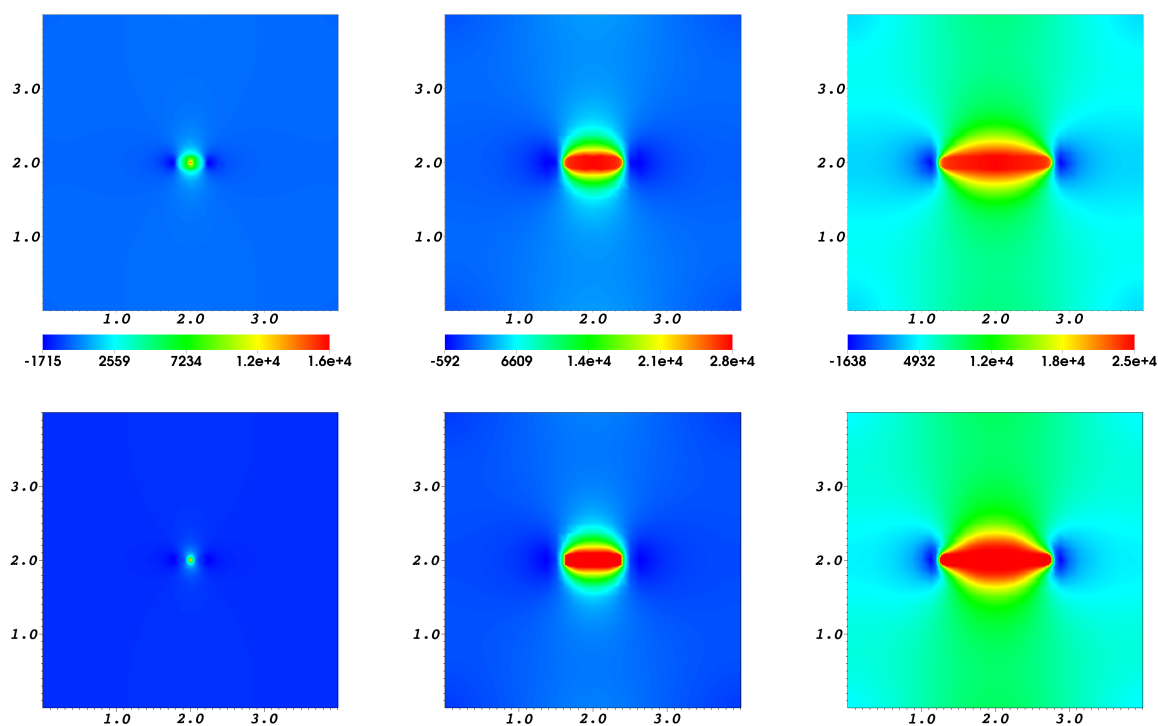

Figure 7: Example 5.1: Pressure distribution at $T=0,0.3,0.5 \mathrm{~s}$ for $m=1$ (Newtonian, top) and $m=0.5$ (quasi-Newtonian, bottom). Using the quasi-Newtonian model for fracture flow the highest pressure is lower than in the Newtonian case and the fracture propagates slower. We recall that polymers that lead to non-Newtonian fluid behavior are mainly intended to carry the proppant and not for the fracture propagation itself [31]. This is one main reason why fracturing is performed in different stages and specifically water is first injected and polymers is included at a later stage (time).

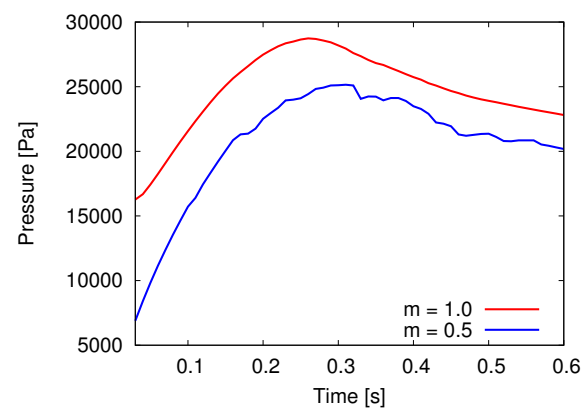

Figure 8: Example 5.1: Maximum pressure evolution (in $P a$ ) versus time (in $s$ ). As long as the crack does not propagate (here until $T=0.25 s$ ) the pressure increases. A pressure decrease follows when the crack evolves. Using the quasi-Newtonian model (here $m=0.5$ ), we observe that the highest pressure is lower than in the Newtonian case.

In the domain $\Lambda=(0 \mathrm{~m}, 4 \mathrm{~m})^{2}$, we set a initial fracture centered at $(2 \mathrm{~m}, 2 \mathrm{~m})$ with radius $r=0.4 \mathrm{~m}$ with thickness of $2 h_{\min }$ as shown in the Figure 10 (a). The mechanical parameters are $G_{c}=1.0 \mathrm{~N} / \mathrm{m}, v=0.2$ and $E=10^{8} \mathrm{~Pa}$ in the homogeneous domain. The fluid is injected at the center of the fracture with the constant volume rate of $q_{F}=200 \mathrm{~m}^{3} / \mathrm{s}$ for point source injection and $q_{L}=0$. The fluid parameters are given as $\eta_{F}=\eta_{R}=1 \times 10^{-3} \mathrm{Ns} / \mathrm{m}^{2}$, $\rho_{R}=\rho_{F}=1000 \mathrm{~kg} / \mathrm{m}^{3}$, with $m=0.9$. Also other parameters are $k_{R}=1 \times 10^{-12}, \mathbf{g}=0, c_{F}=1 \times 10^{-10}$, and the Biot modulus is $M=2.5 \times 10^{8} \mathrm{~Pa}$. Here $\varepsilon=2 h_{\min }, h_{\min }=0.022 \mathrm{~m}$ and the time step is $\delta t=0.01 \mathrm{~s}$, with $T=3 \mathrm{~s}$.

Figure 10 illustrates the concentration of the proppant with the fracture propagation. First, the contour line indicates the fracture $(\varphi=0.8)$ and we observe the different distribution of concentration for different values of $Q=1,10,50,100$ and 250. Larger $Q$ transports the concentration faster to the tip of fracture. In addition, we plot the concentration value over the fracture (line $(2,2)-(3.6,2))$ at Figure 11 (a). The concentration values are affected by the fracture propagation at $Q=10,50,100$, and $Q=250$ cases but not at $Q=1$ case. Note that in case $Q=250$, the speed for proppant transport is so fast that the proppant accumulates at the crack tip. We also observe some 


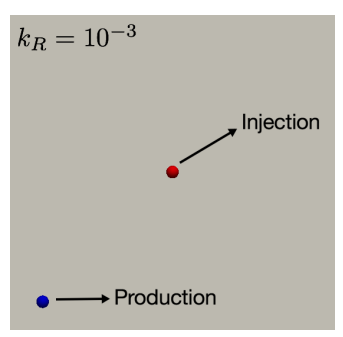

(a) Case i)

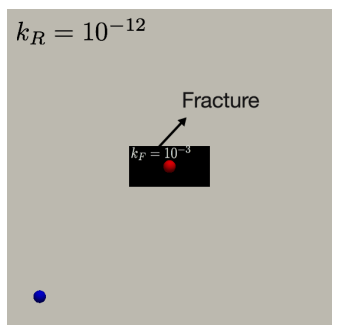

(e) Case ii)

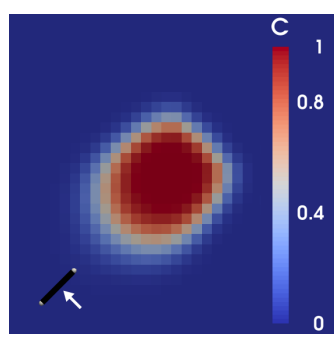

(b) Case i) at $t=10$

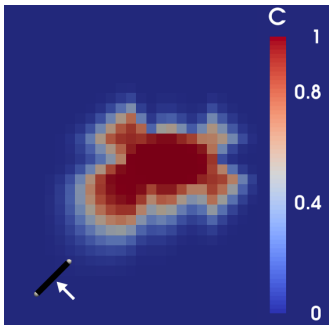

(f) Case ii) at $t=10$

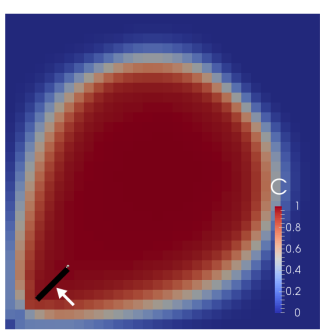

(c) Case i) at $t=1000$

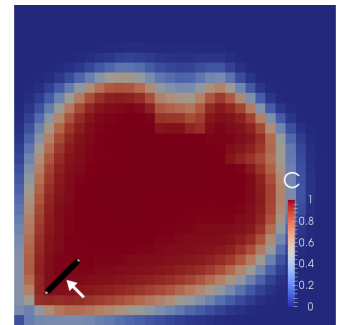

(g) Case ii) at $t=1000$

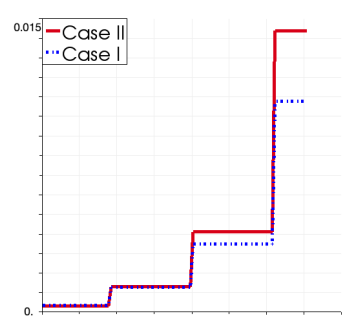

(d) Comparison at $t=10$

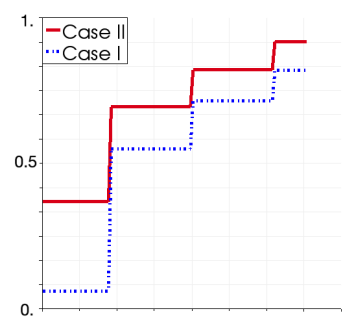

(h) Comparison at $t=1000$

Figure 9: Example 5.2 (a) Setup for case i) with $k_{R}=k_{F}=10^{-3}$ in the domain and for (e) case ii) with the fracture in the middle $\left(k_{F}=10^{-3}\right.$ ) but lower permeability in the reservoir $k_{R}=10^{-12}$. At each time $t=10$ and $t=1000$, (b)-(c) illustrates the concentration values for case i) and (f)-(g) for case ii). We compare the concentration values between each cases over the line $(1,1)-(2,2)$ at $(\mathrm{d})$-(h) and we observe the higher value of concentration near the production for case ii) with the fixed fracture at the middle of the domain.

higher accumulation of proppant in the middle of the fracture since the concentration depends on the crack width and permeability.

However, Figure 11 (b) shows the case with $Q=250$ at $t=3$ applying non-locally conservative (continuous Galerkin) flux to transport the concentration value. We observe non physical phenomena with extremely high values and this is the critical reason to use locally conservative flux.

\subsection{2. $3 D$ setting}

In the three dimensional domain $\Lambda=(0 \mathrm{~m}, 4 \mathrm{~m})^{3}$, the initial penny shape fracture is centered at $(2 \mathrm{~m}, 2 \mathrm{~m}, 2 \mathrm{~m}) \mathrm{on}$ $y=2 \mathrm{~m}$-plane with the radius $r=0.25 \mathrm{~m}$. The fluid is injected at the center of the fracture and all the parameters are the same as in the previous two dimensional example. Here $\varepsilon=2 h_{\min }, h_{\min }=0.054 \mathrm{~m}$ and the time step is $\delta t=0.01 s$.

Figure 12 illustrates each step of the fracture propagation due to the injection of fluid with the transport of concentration. In addition, we vary the $Q$ values for the pressure diffraction system to study the differences. This numerical example highlights the capabilities of our algorithm to handle 3D computations.

\subsection{Two fractures with proppant transport}

In this last example, we predict concentration values in two initial fractures in arbitrary positions propagating due to injection. We also emphasize joining and branching of the fractures with locally refined meshes.

Figure 13 (a) presents the initial setup for the multiple fractures on the locally refined domain $\Lambda=(0 \mathrm{~m}, 4 \mathrm{~m})^{2}$. The right fracture is centered at $(2.25 \mathrm{~m}, 2 \mathrm{~m})$ with length $0.4 \mathrm{~m}$ and the left fracture is centered at $(1.75 \mathrm{~m}, 2.5 \mathrm{~m})$ with length $r=0.5 \mathrm{~m}$. The mechanical parameters are $v=0.2$ and $E=10^{8} \mathrm{~Pa}$ for the homogeneous domain. Here the fluid is injected at the center of the fracture with the volume rate $q_{F}=200 \mathrm{~m}^{3} / \mathrm{s}$. The discretization parameters are $\delta t=0.01 s, \varepsilon=2 h_{\min }$, and $h_{\min }=0.054 \mathrm{~m}$.

Figure 13 illustrates the concentration values with fractures propagating for each time step $n$. Here the red contour line indicates the evolving fractures with the phase field value $\varphi=0.8$, and the proppant concentration is moving inside the fractures with $Q=100$. 


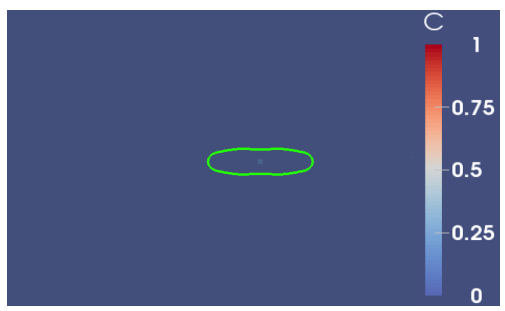

(a) $Q=1$ at $t=0.5$

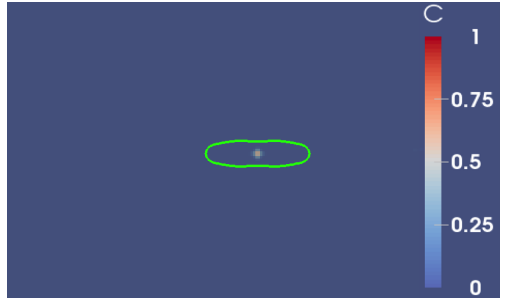

(d) $Q=10$ at $t=0.5$

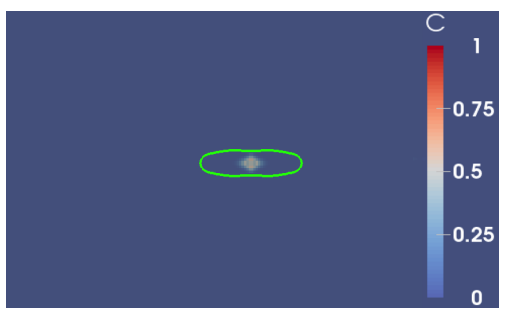

(g) $Q=50$ at $t=0.5$

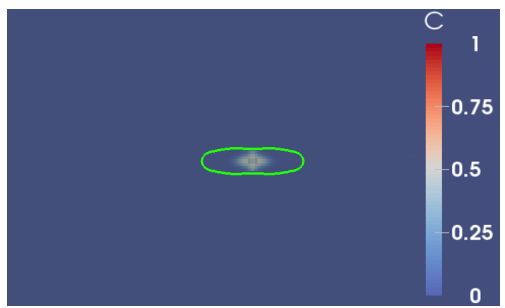

(j) $Q=100$ at $t=0.5$

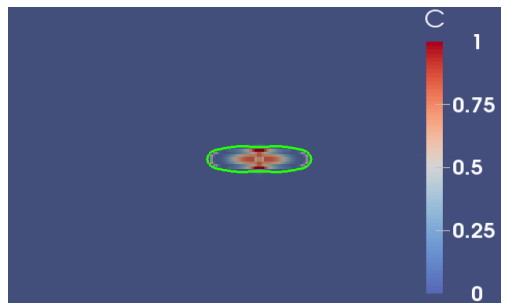

(m) $Q=250$ at $t=0.5$

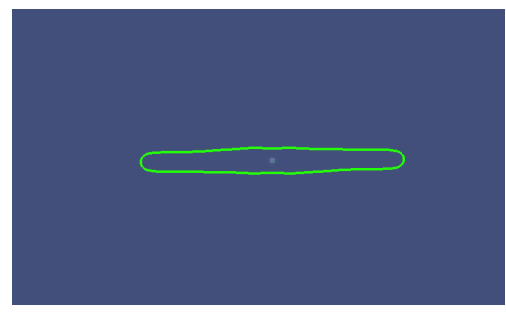

(b) $Q=1$ at $t=1$

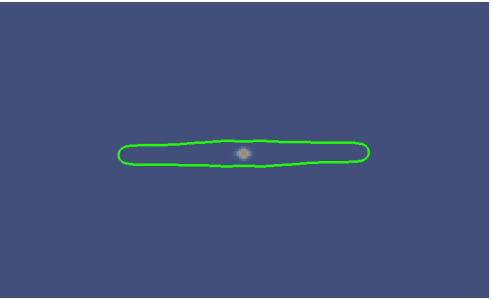

(e) $Q=10$ at $t=1$

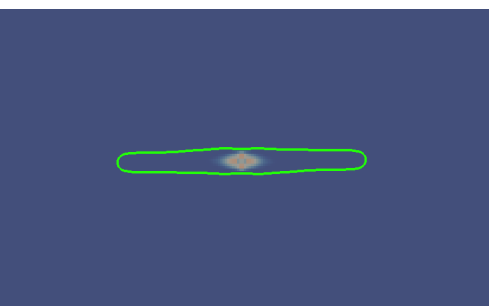

(h) $Q=50$ at $t=1$

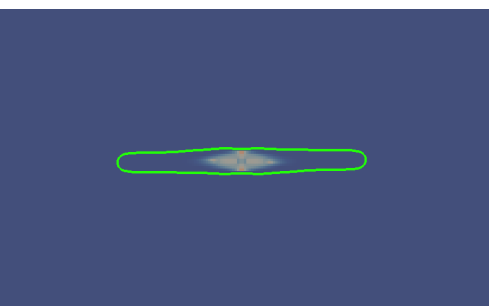

(k) $Q=100$ at $t=1$

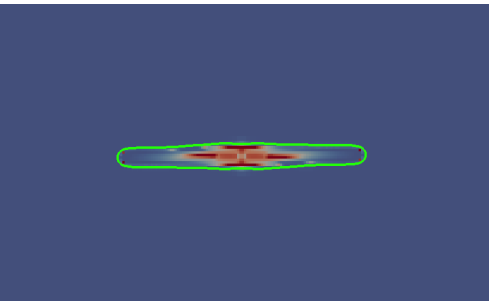

(n) $Q=250$ at $t=1$

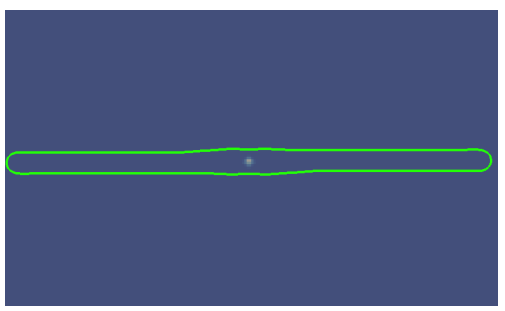

(c) $Q=1$ at $t=3$

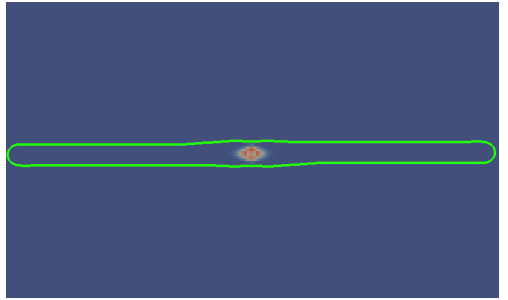

(f) $Q=10$ at $t=3$

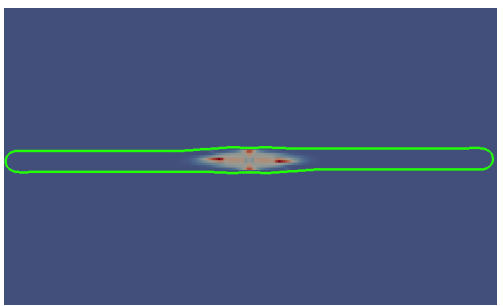

(i) $Q=50$ at $t=3$

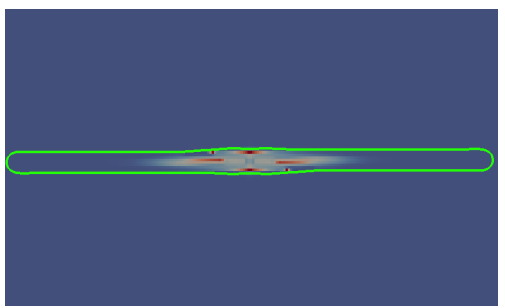

(1) $Q=100$ at $t=3$

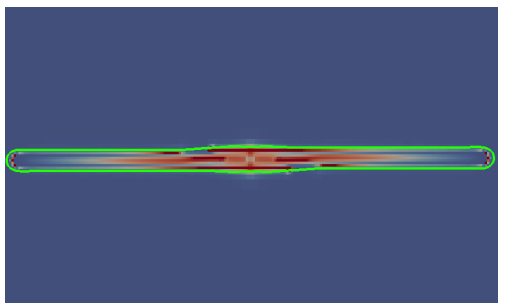

(o) $Q=250$ at $t=3$

Figure 10: Example 5.3.1 For all figures green contour lines indicate the growing fractures with value $\varphi=0.8$. We compare the concentration values $(0 \leq c \leq 1)$ for each time $t=0.5$ (first column), 1 (second column), and 3 (third column) with different values of $Q=1,10,50,100$, and 250 .

\section{Conclusions}

In this contribution, we extended multiphysics phase-field fracture modeling by incorporating a power-law fracture fluid and proppant transport. In order to achieve local mass conservative flow, the enriched Galerkin method has 


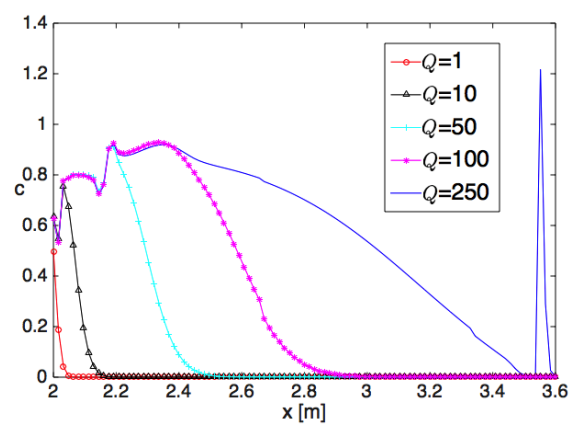

(a)

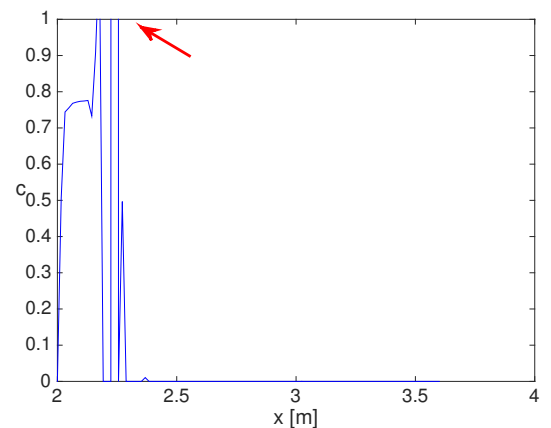

(b)

Figure 11: Example 5.3.1 (a) Comparison of the concentration values for different $Q$ on the line $(2,2)-(3.6,2)$, which is the middle of the fracture at $t=3$ in Figure 10. (b) Illustrates the concentration values when non-locally conservative discretization method (continuous Galerkin) is employed for the flux. The red arrow indicates the concentration values exceeding up to $c=70$. The values are computed on the line $(2,2)-(3.6,2)$ at $t=3$ with $Q=250$.

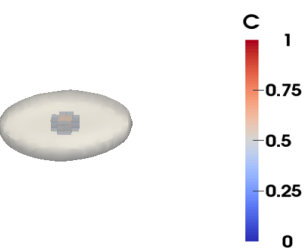

(a) $Q=100 ; n=5$

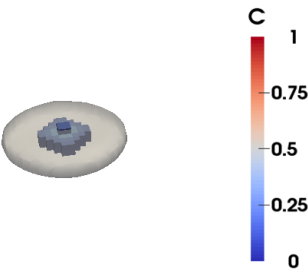

(d) $Q=250 ; n=5$

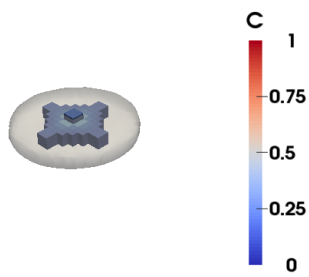

(g) $Q=500 ; n=5$

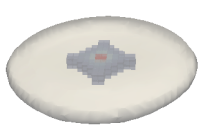

(b) $n=20$

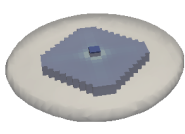

(e) $n=20$

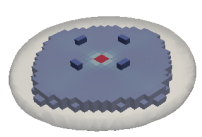

(h) $n=20$

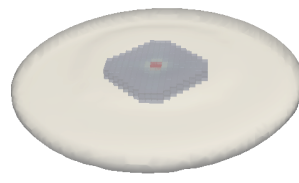

(c) $n=44$

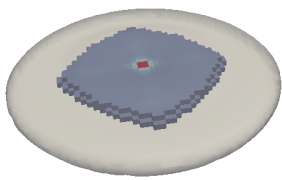

(f) $n=44$

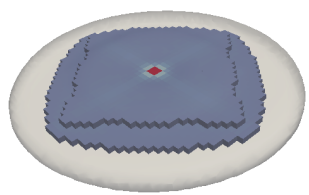

(i) $n=44$

Figure 12: Example 5.3.2 The penny shape fracture propagating for each time $n$ is plotted with phase field contour value for $\varphi=0.8$ outside. Inside the fractures, concentration values along with fracture propagation for different $Q=100,250$, and 500 are illustrated.

been employed. In our numerical examples we considered different scenarios. First, we compare Newtonian versus power-law fracture flow models without solving proppant transport. In the remaining examples, proppant transport is included for quasi-Newtonain fluid. Here, tests in two and three dimensions have been computed where the crack pattern and the concentration distribution have been compared. The proppant transport equation has been restricted 


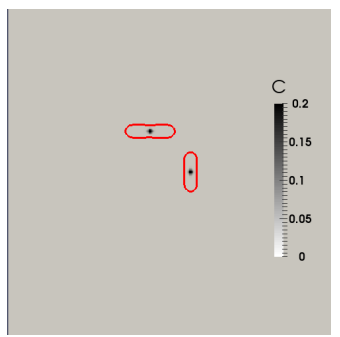

(a) $n=1$

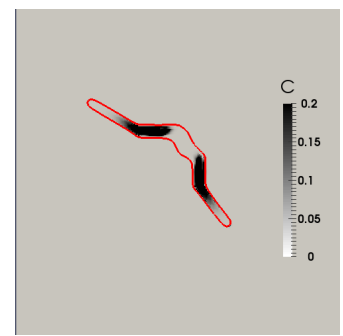

(d) $n=75$

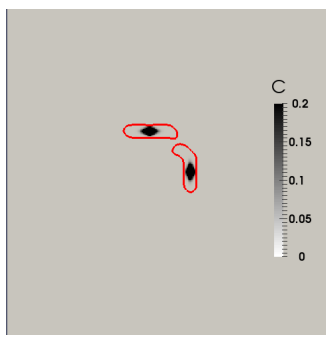

(b) $n=27$

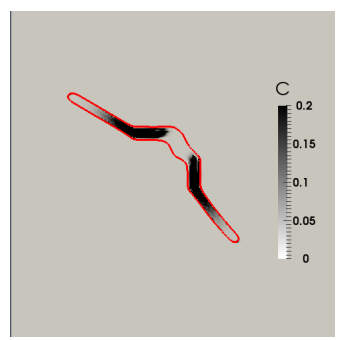

(e) $n=100$

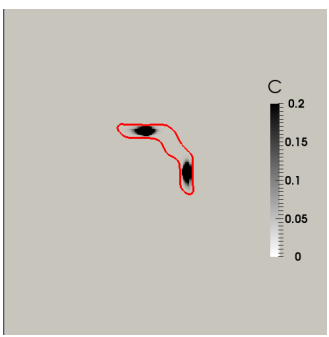

(c) $n=35$

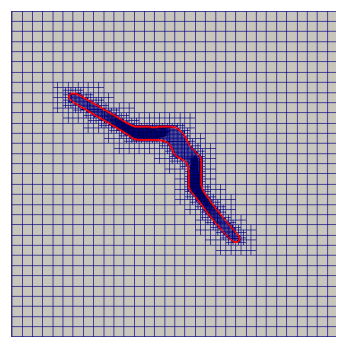

(f) $n=100$

Figure 13: Example 5.4. Concentration values and joining of two fractures for each time step $n$. The solid contour line indicates the fracture $(\varphi=0.8)$ and we emphasize the adaptive mesh refinement at (f).

as a prototype model in this work. Future extensions for including gravitational forces or slip velocity vectors are planned.

\section{Acknowledgments}

All the authors wish to acknowledge partial support by Department of Energy National Energy Technology Laboratory grant DE-FE0023314, Aramco grant UTA 11-000320, and Statoil grant STNO-4502931834. A. Mikelić would like to thank the Institute for Computational Engineering and Science (ICES), UT Austin for hospitality during his visits in February and June 2015. T. Wick was partially supported by the Austrian Academy of Sciences, the Institute for Computational Engineering and Sciences JT Oden fellowship, and the Center for Subsurface Modeling at UT Austin.

\section{Appendix: Nonlinear Darcy's law for a power law in a flat 3D penny shape fracture}

In the appendix we provide a short derivation of the relation between the velocity and the pressure gradient for power law quasi-Newtonian fracture flow. Here, $e(\mathbf{v})$ denotes the rate of the strain tensor, i.e. the symmetrized gradient of the velocity

$$
e(\mathbf{v})=\frac{1}{2}\left(\nabla \mathbf{v}+(\nabla \mathbf{v})^{\tau}\right)
$$

The matrix norm $|\cdot|$ is defined by

$$
|\xi|^{2}=\operatorname{Tr}\left(\xi \xi^{t}\right), \xi \in \mathbb{R}^{9}
$$

The (fracture) viscosity $\eta_{F}^{e f f}$ is assumed to be a power-law function of the microscopic shear strain rate $e(\mathbf{v})$, which is expressed in term of the second invariant of the strain tensor $e_{I I}(\mathbf{v})=\operatorname{Tr} e(\mathbf{v})^{2}$ by

$$
\eta_{F}^{e f f}=\eta_{F} \dot{\gamma}^{m-1}=\eta_{F}|\sqrt{2} e(\mathbf{v})|^{m-1}=\eta_{F}|\sqrt{2} e(\mathbf{v})|^{r-2},
$$

where $\eta_{F}$ is the shear consistency of the fluid, $m$ is the power-law exponent, characterizing the strain rate sensitivity of the fluid, and $r=m+1,1<r<+\infty$. For more details we refer to [10]. 
In the following, the notation and geometry assumptions follow the Appendix of [60]. A flat 3D fracture $\Omega_{C}=C$ is characterized by the ratio ${ }^{1} \varepsilon$ between its characteristic width $H$ in the direction $x_{3}$ and its characteristic horizontal length $L$ in directions $x_{1}$ and $x_{2}$; a sketch is provided in Figure 3 . Here we assume that the crack boundary is given by

$$
x_{3}=h^{(2)}\left(x_{1}, x_{2}, t\right) \quad \text { for } x_{3} \geq 0 \quad \text { and } \quad x_{3}=h^{(1)}\left(x_{1}, x_{2}, t\right) \text { for } x_{3}<0, t \in[0, T] .
$$

Here $h^{(2)}$ and $h^{(1)}$ meet at $x_{3}=0$, the tip region. $\omega$ is a smooth bounded domain in $\mathbb{R}^{2}$ and

$$
\Omega_{C}(t)=\left\{\left(x_{1}, x_{2}, x_{3}\right) \mid h^{(1)}\left(x_{1}, x_{2}, t\right)<x_{3}<h^{(2)}\left(x_{1}, x_{2}, t\right),\left(x_{1}, x_{2}\right) \in \omega\right\}, t \in[0, T] .
$$

The fluid flow in the fracture is described by the incompressible power-law quasi-Newtonian Navier-Stokes equations

$$
\begin{gathered}
\frac{\partial \mathbf{v}}{\partial t}+(\mathbf{v} \cdot \nabla) \mathbf{v}+\frac{1}{\rho_{F}} \nabla p-2 \frac{\eta_{F}}{\rho_{F}} \operatorname{div}\left(|\sqrt{2} e(\mathbf{v})|^{r-2} e(\mathbf{v})\right)=\mathbf{f} \quad \text { in } \Omega_{C}(t) \\
\operatorname{div} \mathbf{v}=q \quad \text { in } \Omega_{C}(t),
\end{gathered}
$$

where $\rho_{F}=\rho_{F}^{0}$ is the fluid density and $v=\eta_{F}|\sqrt{2} e(\mathbf{v})|^{r-2} / \rho_{F}$ is the kinematic viscosity. For simplicity, we assume that the volume force $\mathbf{f}$ is independent of $x_{3}$.

Next, we derive a dimensionless form of the lubrication equation following the classical lubrication theory references (see e.g. [73]). Let $U$ be the characteristic velocity in the directions $x_{1}$ and $x_{2}$ and $\mathbf{R e}=\frac{U L \rho_{F}}{\eta_{F}}$ the Reynolds number. Then we set

$$
\begin{gathered}
\bar{t}=\frac{t}{T_{0}} ; \quad x=\frac{x_{1}}{L}, \quad y=\frac{x_{2}}{L}, \quad z=\frac{x_{3}}{H}, \quad v_{x}^{\varepsilon}=\frac{v_{1}}{U}, \quad v_{y}^{\varepsilon}=\frac{v_{2}}{U}, \quad v_{z}^{\varepsilon}=\frac{v_{3} L}{U H}, \bar{q}=q \frac{L}{U}, \\
\overline{\mathbf{f}}=\mathbf{f} \frac{\rho_{F} H^{r}}{\eta_{F} U^{r-1}}, \quad \bar{p}=\frac{H^{r}}{\eta_{F} L U^{r-1}} p, \quad w^{(j)}(x, y, \bar{t})=\frac{1}{H} h^{(j)}\left(x_{1}, x_{2}, t\right) \quad \text { and } \quad \varepsilon=\frac{H}{L} .
\end{gathered}
$$

The symmetrized velocity gradients transform as follows

$$
\begin{aligned}
& e_{i j}(\mathbf{v})=\frac{1}{2}\left(\frac{\partial v_{i}}{\partial x_{j}}+\frac{\partial v_{j}}{\partial x_{i}}\right)=\frac{U}{L} e_{i j}\left(\mathbf{v}^{\varepsilon}\right), \quad 1 \leq i, j \leq 2 ; \\
& e_{i 3}(\mathbf{v})=\frac{1}{2}\left(\frac{\partial v_{i}}{\partial x_{3}}+\frac{\partial v_{3}}{\partial x_{i}}\right)=\frac{U}{2 H}\left(\partial_{z} v_{i}^{\varepsilon}+\varepsilon^{2} \partial_{x_{i}} v_{z}^{\varepsilon}\right), \quad 1 \leq i \leq 2 ; \\
& e_{33}(\mathbf{v})=\frac{U}{L} \partial_{z} v_{z}^{\varepsilon} .
\end{aligned}
$$

Lemma 15. Let the nonlinear viscosity $\mathcal{S}$ be defined as

$$
\mathcal{S}\left(\left|\partial_{z}\left(v_{x}^{\varepsilon}, v_{y}^{\varepsilon}\right)\right|\right)=\left(\left|\partial_{z} v_{x}^{\varepsilon}\right|^{2}+\left|\partial_{z} v_{y}^{\varepsilon}\right|^{2}\right)^{r / 2-1}
$$

Then we have

$$
v=\frac{\eta_{F}}{\rho_{F}}\left(\frac{U}{H}\right)^{r-2} \mathcal{S}\left(\left|\partial_{z}\left(v_{x}^{\varepsilon}, v_{y}^{\varepsilon}\right)\right|\right)+O\left(\varepsilon^{2}\right) .
$$

\footnotetext{
${ }^{1}$ We note that the $\varepsilon$ used in the Appendix is not related to the phase-field regularization parameter.
} 
For simplicity, we skip all bars and denote the rescaled $\Omega_{C}$ with the same symbol. The system (50)-(51) becomes

$$
\begin{gathered}
\frac{\rho_{F} U^{3-r} H^{r}}{L \eta_{F}}\left(\frac{L}{U T_{0}} \partial_{t} v_{x}^{\varepsilon}+\left(\left(v_{x}^{\varepsilon}, v_{y}^{\varepsilon}, v_{z}^{\varepsilon}\right) \cdot \nabla_{x, y, z}\right) v_{x}^{\varepsilon}\right) \\
=-\partial_{x} p^{\varepsilon}+\partial_{z}\left(\mathcal{S}\left(\left|\partial_{z}\left(v_{x}^{\varepsilon}, v_{y}^{\varepsilon}\right)\right|\right) \partial_{z} v_{x}^{\varepsilon}\right)+f_{x}+O\left(\varepsilon^{2}\right) \quad \text { in } \Omega_{C}(t), \\
\frac{\rho_{F} U^{3-r} H^{r}}{L \eta_{F}}\left(\frac{L}{U T_{0}} \partial_{t} v_{y}^{\varepsilon}+\left(\left(v_{x}^{\varepsilon}, v_{y}^{\varepsilon}, v_{z}^{\varepsilon}\right) \cdot \nabla_{x, y, z}\right) v_{y}^{\varepsilon}\right) \\
=-\partial_{y} p^{\varepsilon}+\partial_{z}\left(\mathcal{S}\left(\left|\partial_{z}\left(v_{x}^{\varepsilon}, v_{y}^{\varepsilon}\right)\right|\right) \partial_{z} v_{y}^{\varepsilon}\right)+f_{y}+O\left(\varepsilon^{2}\right) \quad \text { in } \Omega_{C}(t), \\
\frac{\rho_{F} U^{3-r} H^{r-1}}{\eta_{F}}\left(\partial_{t} v_{z}^{\varepsilon}+\left(\left(v_{x}^{\varepsilon}, v_{y}^{\varepsilon}, v_{z}^{\varepsilon}\right) \cdot \nabla_{x, y, z}\right) v_{z}^{\varepsilon}\right)=-\frac{1}{\varepsilon^{2}} \partial_{z} p^{\varepsilon}+\frac{1}{\varepsilon} f_{z}+ \\
2 \partial_{z}\left(\mathcal{S}\left(\left|\partial_{z}\left(v_{x}^{\varepsilon}, v_{y}^{\varepsilon}\right)\right|\right) \partial_{z} v_{z}^{\varepsilon}\right)+\operatorname{div}_{x, y}\left(\mathcal{S}\left(\left|\partial_{z}\left(v_{x}^{\varepsilon}, v_{y}^{\varepsilon}\right)\right|\right) \partial_{z}\left(v_{x}^{\varepsilon}, v_{y}^{\varepsilon}\right)\right)+O(\varepsilon) \quad \text { in } \Omega_{C}(t), \\
\partial_{x} v_{x}^{\varepsilon}+\partial_{y} v_{y}^{\varepsilon}+\partial_{z} v_{z}^{\varepsilon}=q+O\left(\varepsilon^{2}\right) \quad \text { in } \Omega_{C}(t) .
\end{gathered}
$$

We assume $\frac{\rho_{F} U^{3-r} H^{r}}{L \eta_{F}} \ll 1$, and $\frac{L}{T_{0} U} \ll 1$ and neglect the inertia term at the main order.

\subsection{Incorporating boundary conditions}

Concerning the boundary conditions, we recall that the unit normal $\mathbf{n}$ and tangential vectors $\tau^{(j)}, j=1,2$ can be expressed in the form

$$
\mathbf{n}=\frac{-\varepsilon \partial_{x} w \mathbf{e}^{1}-\varepsilon \partial_{y} w \mathbf{e}^{2}+\mathbf{e}^{3}}{\sqrt{1+\varepsilon^{2}\left|\nabla_{x, y} w\right|^{2}}} ; \quad \tau^{(1)}=\frac{\mathbf{e}^{1}+\varepsilon \partial_{x} w \mathbf{e}^{3}}{\sqrt{1+\varepsilon^{2}\left|\partial_{x} w\right|^{2}}} ; \quad \tau^{(2)}=\frac{\mathbf{e}^{2}+\varepsilon \partial_{y} w \mathbf{e}^{3}}{\sqrt{1+\varepsilon^{2}\left|\partial_{y} w\right|^{2}}} .
$$

Then for $\mathbf{v}^{\varepsilon}=\left(v_{x}^{\varepsilon}, v_{y}^{\varepsilon}, v_{z}^{\varepsilon}\right)$ we have

$$
\begin{gathered}
\mathbf{v}^{\varepsilon} \cdot \mathbf{n}=\varepsilon \frac{-\partial_{x} w v_{x}^{\varepsilon}-\partial_{y} w v_{y}^{\varepsilon}+v_{z}^{\varepsilon}}{\sqrt{1+\varepsilon^{2}\left|\nabla_{x, y} w\right|^{2}}}=\varepsilon\left(v_{z}^{\varepsilon}-\nabla_{x, y} w \cdot\left(v_{x}^{\varepsilon}, v_{y}^{\varepsilon}\right)\right)+O\left(\varepsilon^{3}\right) \\
\mathbf{v}^{\varepsilon} \cdot \tau^{(1)}=\frac{v_{x}^{\varepsilon}+\varepsilon^{2} \partial_{x} w v_{z}^{\varepsilon}}{\sqrt{1+\varepsilon^{2}\left|\partial_{x} w\right|^{2}}}=v_{x}^{\varepsilon}+\varepsilon^{2}\left(\partial_{x} w v_{z}^{\varepsilon}-\frac{v_{x}^{\varepsilon}}{2}\left(\partial_{x} w\right)^{2}\right)+O\left(\varepsilon^{4}\right)
\end{gathered}
$$

and analogously for $\mathbf{v}^{\varepsilon} \cdot \tau^{(2)}$.

We assume that the velocity in the fracture is larger than in the surrounding porous medium. Hence we have at $z=w(x, y, t)$ :

$$
0=\mathbf{v}^{\varepsilon} \cdot \tau^{(1)}=v_{x}^{\varepsilon}+\varepsilon^{2}\left(\partial_{x} w v_{z}^{\varepsilon}-\frac{v_{x}^{\varepsilon}}{2}\left(\partial_{x} w\right)^{2}\right)+O\left(\varepsilon^{4}\right)
$$

and an analogous expression for $\mathbf{v}^{\varepsilon} \cdot \tau^{(2)}$. In normal direction we must impose the kinematic boundary condition:

$$
\frac{D h}{D t}=-v_{\text {out }} \text {. }
$$

The term $v_{\text {out }}$ is used to calculate the previously introduced leak-off $q_{L}$. We then obtain

$$
\partial_{t} h+\left(v_{1}, v_{2}\right) \cdot \nabla_{x_{1}, x_{2}} h-v_{3}=-v_{\text {out }} \quad \text { on } \quad x_{3}=h\left(x_{1}, x_{2}, t\right),
$$

and in the dimensionless form we have

$$
\frac{L}{T_{0} U} \partial_{t} w+\left(v_{x}^{\varepsilon}, v_{y}^{\varepsilon}\right) \cdot \nabla_{x, y} w-v_{z}^{\varepsilon}=-v_{L O} \quad \text { on } \quad z=w(x, y, t)
$$

where $v_{\text {out }}=U \varepsilon v_{L O}$.

Remark 16. An alternative to the zero tangential velocity condition $\sqrt{58}$ is to impose the Beavers and Joseph slip condition on the moving boundary:

$$
M\left[\begin{array}{c}
\mathbf{v}^{\varepsilon} \cdot \tau^{(1)} \\
\mathbf{v}^{\varepsilon} \cdot \tau^{(2)}
\end{array}\right]=\left[\begin{array}{l}
v\left(\left|e\left(\mathbf{v}^{\varepsilon}\right)\right|\right) e\left(\mathbf{v}^{\varepsilon}\right) \mathbf{n} \cdot \tau^{(1)} \\
v\left(\left|e\left(\mathbf{v}^{\varepsilon}\right)\right|\right) e\left(\mathbf{v}^{\varepsilon}\right) \mathbf{n} \cdot \tau^{(1)}
\end{array}\right]
$$

where $M$ is the (symmetric) slip matrix. The slip law [60) was introduced in [9]. 


\subsection{The lubrication approximation}

Following classical expansions from lubrication theory, we expand the velocities and the pressure as follows

$$
\begin{aligned}
& v_{x}^{\varepsilon}=v_{x}^{0}+\varepsilon^{2} v_{x}^{1}+O\left(\varepsilon^{4}\right) ; \quad v_{y}^{\varepsilon}=v_{y}^{0}+\varepsilon^{2} v_{y}^{1}+O\left(\varepsilon^{4}\right) ; \quad v_{z}^{\varepsilon}=v_{z}^{0}+\varepsilon^{2} v_{z}^{1}+O\left(\varepsilon^{4}\right) ; \\
& p^{\varepsilon}=p^{0}+\varepsilon^{2} p^{1}+O\left(\varepsilon^{4}\right) ; \\
& w= \begin{cases}w^{(2)}(x, y, t) & \text { for } \quad z \geq 0, \text { (the upper surface) } \\
w^{(1)}(x, y, t) & \text { for } \quad z<0 \text { (the lower surface). }\end{cases}
\end{aligned}
$$

Inserting (61)-63) into 547- (57) yields at the order $O(1)$ in $\Omega_{C}$ :

$$
\begin{gathered}
-\partial_{x} p^{0}+f_{x}+\partial_{z}\left(\mathcal{S}\left(\left|\partial_{z}\left(v_{x}^{0}, v_{y}^{0}\right)\right|\right) \partial_{z} v_{x}^{0}\right)=0 ;-\partial_{y} p^{0}+f_{y}+\partial_{z}\left(\mathcal{S}\left(\left|\partial_{z}\left(v_{x}^{0}, v_{y}^{0}\right)\right|\right) \partial_{z} v_{y}^{0}\right)=0 ; \\
-\partial_{z} p^{0}=0 ; \quad \partial_{x} v_{x}^{0}+\partial_{y} v_{y}^{0}+\partial_{z} v_{z}^{0}=q ; \\
v_{x}^{0}(x, y, w, t)=0=v_{y}^{0}(x, y, w, t) .
\end{gathered}
$$

Following [73], we use 647-666 to calculate $v_{x}^{0}, v_{y}^{0}$ and obtain a PDE for $p^{0}$. However, presence of the nonlinear viscosity $\mathcal{S}$ complicates the task and we start with an viscosity expression as a function of $\left|\left(f_{x}, f_{y}\right)-\nabla_{x, y} p^{0}\right|$ :

Proposition 17. The nonlinear viscosity $\mathcal{S}$, defined by (52), satisfies

$$
\mathcal{S}\left(\left|\partial_{z}\left(v_{x}^{0}, v_{y}^{0}\right)\right|\right)=\left|\left(f_{x}, f_{y}\right)-\nabla_{x, y} p^{0}\right|^{(r-2) /(r-1)}\left|z-\frac{w^{(1)}+w^{(2)}}{2}\right|^{(r-2) /(r-1)} .
$$

Proof. The first equation in 65 yields $p^{0}=p^{0}(x, y, t)$ (the geostrophic approximation). Next we integrate the equations in (64) to get

$$
\begin{aligned}
& \mathcal{S}\left(\left|\partial_{z}\left(v_{x}^{0}, v_{y}^{0}\right)\right|\right) \partial_{z} v_{x}^{0}=A_{x}(x, y, t)+z\left(\partial_{x} p^{0}-f_{x}\right), \\
& \mathcal{S}\left(\left|\partial_{z}\left(v_{x}^{0}, v_{y}^{0}\right)\right|\right) \partial_{z} v_{y}^{0}=A_{y}(x, y, t)+z\left(\partial_{y} p^{0}-f_{y}\right) .
\end{aligned}
$$

Formulas (68)-(69) yield

$$
\mathcal{S}\left(\left|\partial_{z}\left(v_{x}^{0}, v_{y}^{0}\right)\right|\right)=\mathcal{S}(z)=\left(\left(A_{x}+z\left(\partial_{x} p^{0}-f_{x}\right)\right)^{2}+\left(A_{y}+z\left(\partial_{y} p^{0}-f_{y}\right)\right)^{2}\right)^{(r-2) /(2 r-2)} .
$$

It remains to calculate $A_{x}$ and $A_{y}$. We first integrate $\sqrt{68}$ - $-\sqrt{69}$ between $w^{(1)}$ and $z$ and obtain the following expressions for the velocity components:

$$
\begin{aligned}
& v_{x}^{0}(z)=A_{x} \int_{w^{(1)}}^{z} \frac{d \xi}{\mathcal{S}(\xi)}+\left(\partial_{x} p^{0}-f_{x}\right) \int_{w^{(1)}}^{z} \frac{\xi d \xi}{\mathcal{S}(\xi)}, \\
& v_{y}^{0}(z)=A_{y} \int_{w^{(1)}}^{z} \frac{d \xi}{\mathcal{S}(\xi)}+\left(\partial_{y} p^{0}-f_{y}\right) \int_{w^{(1)}}^{z} \frac{\xi d \xi}{\mathcal{S}(\xi)} .
\end{aligned}
$$

Let us introduce the following abbreviations

$$
a=a(x, y, t)=\int_{w^{(1)}}^{w^{(2)}} \frac{d \xi}{\mathcal{S}(\xi)} \quad \text { and } \quad b=b(x, y, t)=\int_{w^{(1)}}^{w^{(2)}} \frac{\xi d \xi}{\mathcal{S}(\xi)} .
$$

Then we have

$$
A_{x}=-\left(\partial_{x} p^{0}-f_{x}\right) \frac{b}{a} \quad \text { and } \quad A_{y}=-\left(\partial_{y} p^{0}-f_{y}\right) \frac{b}{a},
$$

which yields

$$
\mathcal{S}(z)=\left|\left(f_{x}, f_{y}\right)-\nabla_{x, y} p^{0}\right|^{(r-2) /(r-1)}\left|z-\frac{b}{a}\right|^{(r-2) /(r-1)} .
$$


Next,

$$
v_{x}^{0}=\left(\partial_{x} p^{0}-f_{x}\right) \int_{w^{(1)}}^{z} \frac{\left(\xi-\frac{b}{a}\right) d \xi}{\mathcal{S}(\xi)}=\frac{r-1}{r}\left(\partial_{x} p^{0}-f_{x}\right)\left|\left(f_{x}, f_{y}\right)-\nabla_{x, y} p^{0}\right|^{(2-r) /(r-1)}\left(\left|z-\frac{b}{a}\right|^{r /(r-1)}-\left|w^{(1)}-\frac{b}{a}\right|^{r /(r-1)}\right) .
$$

The boundary condition $v_{x}^{0}\left(w^{(2)}\right)=0$ yields $\frac{b}{a}=\frac{w^{(1)}+w^{(2)}}{2}$ and we have

$$
\begin{gathered}
v_{x}^{0}=\frac{r-1}{r}\left(\partial_{x} p^{0}-f_{x}\right)\left|\left(f_{x}, f_{y}\right)-\nabla_{x, y} p^{0}\right|^{(2-r) /(r-1)}\left(\left|z-\frac{w^{(1)}+w^{(2)}}{2}\right|^{r /(r-1)}-\left|\frac{w^{(1)}-w^{(2)}}{2}\right|^{r /(r-1)}\right), \\
v_{y}^{0}=\frac{r-1}{r}\left(\partial_{y} p^{0}-f_{y}\right)\left|\left(f_{x}, f_{y}\right)-\nabla_{x, y} p^{0}\right|^{(2-r) /(r-1)}\left(\left|z-\frac{w^{(1)}+w^{(2)}}{2}\right|^{r /(r-1)}-\left|\frac{w^{(1)}-w^{(2)}}{2}\right|^{r /(r-1)}\right), \\
\mathcal{S}(z)=\left|\left(f_{x}, f_{y}\right)-\nabla_{x, y} p^{0}\right|^{(r-2) /(r-1)}\left|z-\frac{w^{(1)}+w^{(2)}}{2}\right|^{(r-2) /(r-1)} .
\end{gathered}
$$

Remark 18. If the zero tangential velocity condition (58) is replaced by Beavers and Joseph slip condition (60) at the moving boundary, then the conditions (66) have to be replaced by

$$
\left.M^{0}\left[\begin{array}{c}
v_{x}^{0} \\
v_{y}^{0}
\end{array}\right]\right|_{z=w^{(j)}}=\left.\mathcal{S}\left(w^{(j)}\right) \partial_{z}\left[\begin{array}{c}
v_{x}^{0} \\
v_{y}^{0}
\end{array}\right]\right|_{z=w^{(j)}}, j=1,2,
$$

where $M=\frac{\eta_{F}}{H \rho_{F}}\left(\frac{U}{H}\right)^{r-2} M^{0}$ and $M^{0}$ is the (symmetric) dimensionless slip matrix. Considerations analogous to the above ones give us expressions for $\left(v_{x}^{0}, v_{y}^{0}\right)$.

With these preparations, we are ready to calculate the corresponding nonlinear permeability. First let us identify the coefficient in the relations linking $\left(v_{x}^{0}, v_{y}^{0}\right)$ and $\nabla_{x, y} p^{0}$. Let $r^{*}=r /(r-1)$ be the conjugate exponent to $r$.

$$
\begin{aligned}
& K(z)=-\frac{r-1}{r}\left|\left(f_{x}, f_{y}\right)-\nabla_{x, y} p^{0}\right|^{(2-r) /(r-1)}\left(\left|z-\frac{w^{(1)}+w^{(2)}}{2}\right|^{r /(r-1)}-\left|\frac{w^{(1)}-w^{(2)}}{2}\right|^{r /(r-1)}\right) \\
& =\tilde{K}(z)\left|\left(f_{x}, f_{y}\right)-\nabla_{x, y} p^{0}\right|^{(2-r) /(r-1)}, \\
& v_{x}^{0}=-K(z)\left(\partial_{x} p^{0}-f_{x}\right), \quad v_{y}^{0}=-K(z)\left(\partial_{y} p^{0}-f_{y}\right), \\
& \text { For } z \geq\left(w^{(1)}+w^{(2)}\right) / 2, K_{e}(z)=\int_{z}^{w^{(2)}} K(s) d s=\frac{\left|\left(f_{x}, f_{y}\right)-\nabla_{x, y} p^{0}\right|^{(2-r) /(r-1)}}{r^{*}\left(1+r^{*}\right) 2^{1+r^{*}}} \times \\
& \left(\left(w^{(2)}-w^{(1)}\right)^{r^{*}}\left(-2 z+w^{(2)}+w^{(1)}+2 r^{*}\left(w^{(2)}-z\right)\right)+\left(2 z-w^{(2)}-w^{(1)}\right)^{1+r^{*}}\right), \\
& \text { For } z \leq\left(w^{(1)}+w^{(2)}\right) / 2, K_{e}(z)=\int_{z}^{w^{(2)}} K(s) d s=\frac{\left|\left(f_{x}, f_{y}\right)-\nabla_{x, y} p^{0}\right|^{(2-r) /(r-1)}}{r^{*}\left(1+r^{*}\right) 2^{1+r^{*}}} \times \\
& \left(r^{*}\left(w^{(2)}-w^{(1)}\right)^{1+r^{*}}+\left(w^{(1)}+w^{(2)}-2 z\right)\left(\left(1+r^{*}\right)\left(w^{(2)}-w^{(1)}\right)^{r^{*}}-\left(w^{(1)}+w^{(2)}-2 z\right)^{r^{*}}\right)\right), \\
& K_{e}(z)=\tilde{K}_{e}(z)\left|\left(f_{x}, f_{y}\right)-\nabla_{x, y} p^{0}\right|^{(2-r) /(r-1)}, \\
& K^{a b s}=K_{e}\left(w^{(1)}\right)=\frac{\left(w^{(2)}-w^{(1)}\right)^{1+r^{*}}}{\left(1+r^{*}\right) 2^{r^{*}}}\left|\left(f_{x}, f_{y}\right)-\nabla_{x, y} p^{0}\right|^{(2-r) /(r-1)}=\tilde{K}^{a b s}\left|\left(f_{x}, f_{y}\right)-\nabla_{x, y} p^{0}\right|^{r^{*}-2} .
\end{aligned}
$$

Next we reconsider the the kinematic boundary condition 59. . Using expansions 617 and boundary condition 66 yield

$$
\frac{L}{T_{0} U} \partial_{t} w^{(j)}=v_{z}^{0}\left(x, y, w^{(j)}\right)-v_{L O}^{(j)}, \quad j=1,2
$$


The incompressibility condition from 65 allows to calculate $v_{z}^{0}$ :

$$
\begin{gathered}
\partial_{z} v_{z}^{0}=q-\partial_{x} v_{x}^{0}-\partial_{y} v_{y}^{0}=\tilde{K}(z) \operatorname{div}_{x, y}\left(\left|\left(f_{x}, f_{y}\right)-\nabla_{x, y} p^{0}\right|^{(2-r) /(r-1)}\left(\nabla_{x, y} p^{0}-\left(f_{x}, f_{y}\right)\right)\right)+ \\
\left|\left(f_{x}, f_{y}\right)-\nabla_{x, y} p^{0}\right|^{(2-r) /(r-1)}\left(\nabla_{x, y} p^{0}-\left(f_{x}, f_{y}\right)\right) \cdot \nabla_{x, y} \tilde{K}(z)+q .
\end{gathered}
$$

Integration between $z$ and $w^{(2)}$ in $z$ variable and using $(86)$ yield

$$
\begin{gathered}
v_{z}^{0}=\frac{L}{T_{0} U} \partial_{t} w^{(2)}+v_{L O}^{(2)}-\tilde{K}_{e}(z) \operatorname{div}_{x, y}\left(\left|\left(f_{x}, f_{y}\right)-\nabla_{x, y} p^{0}\right|^{(2-r) /(r-1)}\left(\nabla_{x, y} p^{0}-\left(f_{x}, f_{y}\right)\right)\right)- \\
\left|\left(f_{x}, f_{y}\right)-\nabla_{x, y} p^{0}\right|^{(2-r) /(r-1)}\left(\nabla_{x, y} p^{0}-\left(f_{x}, f_{y}\right)\right) \cdot \nabla_{x, y} \tilde{K}_{e}(z)+\int_{z}^{w^{(2)}} q d \xi .
\end{gathered}
$$

If we take $z=w^{(1)}$, then we arrive at the Reynolds lubrication equation for a power-law fluid:

$$
\frac{L}{T_{0} U} \partial_{t}\left(w^{(2)}-w^{(1)}\right)+v_{L O}^{(2)}-v_{L O}^{(1)}+\int_{w^{(1)}}^{w^{(2)}} q d \xi=\operatorname{div}_{x, y}\left(\tilde{K}^{a b s}\left|\left(f_{x}, f_{y}\right)-\nabla_{x, y} p^{0}\right|^{r^{*}-2}\left(\nabla_{x, y} p^{0}-\left(f_{x}, f_{y}\right)\right)\right) .
$$

The equation $(88)$ is the Reynolds lubrication equation (see [10] and [18]), which is a generalization of the classical Reynolds equation for a Newtonian fluid (see [73]). We note that this expansion is not valid in the fracture tip region and that the Navier-Stokes equation do not simplify there.

Remark 19. For the rigorous mathematical justification of the validity of equation (88) we refer to [57], where convergence of $\mathbf{v}^{\varepsilon}$ to $\mathbf{v}^{0}$ was shown.

Remark 20. Let $c_{F}$ be the proppant compressibility. If $T_{0} \leq c_{F} \eta_{F} \varepsilon^{-2}(U / H)^{r-2}$, then the incompressibility condition should be replaced by the continuity equation for a slightly compressible fluid. It would imply only a slight modification of equation (88). Namely, in such setting one has to add the term $\left(w^{(2)}-w^{(1)}\right) \kappa \partial_{t} p^{0}$ to the left hand side of equation (88). The same modification applies to the equations which follow.

\subsection{Calculation of the nonlinear permeability}

In order to have a Darcy-type law for the effective flow, we still miss an equation for $v_{z}^{0}$.

$$
\begin{aligned}
& \operatorname{div}_{x, y}\left(\left|\left(f_{x}, f_{y}\right)-\nabla_{x, y} p^{0}\right|^{r^{*}-2}\left(\nabla_{x, y} p^{0}-\left(f_{x}, f_{y}\right)\right)\right) \\
= & \frac{1}{\tilde{K}^{a b s}}\left\{\frac{L}{T_{0} U} \partial_{t}\left(w^{(2)}-w^{(1)}\right)+v_{L O}^{(2)}-v_{L O}^{(1)}+\int_{w^{(1)}}^{w^{(2)}} q d \xi\right\}-\left|\left(f_{x}, f_{y}\right)-\nabla_{x, y} p^{0}\right|^{r^{*}-2}\left(\nabla_{x, y} p^{0}-\left(f_{x}, f_{y}\right)\right) \cdot \nabla_{x, y} \log \tilde{K}^{a b s .} .
\end{aligned}
$$

We introduce equation 897 into equation 87 and get

$$
\begin{aligned}
v_{z}^{0}(x, y, t)=\tilde{K}_{e}(z)\left|\left(f_{x}, f_{y}\right)-\nabla_{x, y} p^{0}\right|^{r^{*}-2} \nabla_{x, y} \log \frac{\tilde{K}^{a b s}}{\tilde{K}_{e}(z)} \cdot\left(\nabla_{x, y} p^{0}-\left(f_{x}, f_{y}\right)\right)+ & \\
& \left(1-\frac{\tilde{K}_{e}(z)}{\tilde{K}^{a b s}}\right)\left(\frac{L}{T_{0} U} \partial_{t} w^{(2)}+v_{L O}^{(2)}+\int_{w^{(1)}}^{w^{(2)}} q d \xi\right)+\frac{\tilde{K}_{e}(z)}{\tilde{K}^{a b s}}\left(\frac{L}{T_{0} U} \partial_{t} w^{(1)}+v_{L O}^{(1)}\right) .
\end{aligned}
$$

Hence we got the following Darcy's law

$$
\begin{aligned}
{\left[\begin{array}{c}
v_{x}^{0} \\
v_{y}^{0} \\
v_{z}^{0}
\end{array}\right]=- } & {\left[\begin{array}{ccc}
\tilde{K}(z) & 0 & -\tilde{K}_{e}(z) \partial_{x} \log \frac{\tilde{K}^{a b s}}{\tilde{K}_{e}(z)} \\
0 & \tilde{K}(z) & -\tilde{K}_{e}(z) \partial_{y} \log \frac{\tilde{\tilde{K}}_{e}^{a b s}}{\tilde{K}_{e}(z)} \\
-\tilde{K}_{e}(z) \partial_{x} \log \frac{\tilde{K}_{\tilde{K}}^{a b s}}{\tilde{K}_{e}(z)} & -\tilde{K}_{e}(z) \partial_{y} \log \frac{\tilde{K}^{a b s}}{\tilde{K}_{e}(z)} & 0
\end{array}\right] \frac{\left(\nabla_{x, y} p^{0}-\left(f_{x}, f_{y}\right)\right)}{\left|\left(f_{x}, f_{y}\right)-\nabla_{x, y} p^{0}\right|^{2-r^{*}}} } \\
& +\left[\begin{array}{c}
0 \\
0 \\
\left(1-\frac{\tilde{K}_{e}(z)}{\tilde{K}^{a b s}}\right)\left(\frac{L}{T_{0} U} \partial_{t} w^{(2)}+v_{L O}^{(2)}+\int_{w^{(1)}}^{w^{(2)}} q d \xi\right)+\frac{\tilde{K}_{e}(z)}{\tilde{K}^{a b s}}\left(\frac{L}{T_{0} U} \partial_{t} w^{(1)}+v_{L O}^{(1)}\right)
\end{array}\right] .
\end{aligned}
$$


Darcy's law 91 is supplemented by the equation $\partial_{z} p^{0}=0$. In fact the dependence on $z$ for $\partial_{z}\left(p^{0}-\varepsilon z f_{z}\right)$ comes only at order $O\left(\varepsilon^{2}\right)$. Hence, since Darcy's law 91 , is valid at order $O\left(\varepsilon^{2}\right)$, we can include the equation $\partial_{z} p^{0}=0$ into it by putting an $O\left(\varepsilon^{-2}\right)$ coefficient at the place $(3,3)$ in the permeability. This approach yields

$$
\begin{aligned}
& {\left[\begin{array}{l}
v_{x}^{0} \\
v_{y}^{0} \\
v_{z}^{0}
\end{array}\right]=-\left[\begin{array}{ccc}
\tilde{K} & 0 & -\varepsilon \tilde{K}_{e} \partial_{x} \log \frac{\tilde{K}^{a b s}}{\tilde{K}_{e}} \\
0 & \tilde{K} & -\varepsilon \tilde{K}_{e} \partial_{y} \log \frac{\tilde{K}^{a b s}}{\tilde{K}_{e}} \\
-\tilde{K}_{e} \partial_{x} \log \frac{\tilde{K}^{a b s}}{\tilde{K}_{e}} & -\tilde{K}_{e} \partial_{y} \log \frac{\tilde{K}^{a b s}}{\tilde{K}_{e}} & \frac{\tilde{K}}{\varepsilon}
\end{array}\right] \frac{\left(\left(\nabla_{x, y} p^{0}, \varepsilon^{-1} \partial_{z} p^{0}\right)-\left(f_{x}, f_{y}, f_{z}\right)\right)}{\left|\left(f_{x}, f_{y}, f_{z}\right)-\left(\nabla_{x, y} p^{0}, \varepsilon^{-1} \partial_{z} p^{0}\right)\right|^{2-r^{*}}}} \\
& +\left[\begin{array}{c}
0 \\
0 \\
\left(1-\frac{\tilde{K}_{\tilde{e}}(z)}{\tilde{K}^{a b s}}\right)\left(\frac{L}{T_{0} U} \partial_{t} w^{(2)}+v_{L O}^{(2)}+\int_{w^{(1)}}^{w^{(2)}} q d \xi\right)+\frac{\tilde{K}_{e}(z)}{\tilde{K}^{a b s}}\left(\frac{L}{T_{0} U} \partial_{t} w^{(1)}+v_{L O}^{(1)}\right)
\end{array}\right],
\end{aligned}
$$

implying

$$
\mathbf{v}^{0}=-K\left|\left(\nabla_{x, y} p^{0}, \varepsilon^{-1} \partial_{z} p^{0}\right)-\left(f_{x}, f_{y}, f_{z}\right)\right|^{r^{*}-2}\left(\left(\nabla_{x, y} p^{0}, \varepsilon^{-1} \partial_{z} p^{0}\right)-\left(f_{x}, f_{y}, f_{z}\right)\right)+\mathbf{A}+O(\varepsilon),
$$

with

$$
\begin{gathered}
K=\left[\begin{array}{ccc}
\tilde{K}(z) & 0 & -\varepsilon \tilde{K}_{e}(z) \partial_{x} \log \frac{\tilde{K}^{a b s}}{\tilde{K}_{e}(z)} \\
0 & \tilde{K}(z) & -\varepsilon \tilde{K}_{e}(z) \partial_{y} \log \frac{\tilde{K}^{a b s}}{\tilde{K}_{e}(z)} \\
-\tilde{K}_{e}(z) \partial_{x} \log \frac{\tilde{K}^{a b s}}{\tilde{K}_{e}(z)} & -\tilde{K}_{e}(z) \partial_{y} \log \frac{\tilde{K}^{a b s}}{\tilde{K}_{e}(z)} & \frac{\tilde{K}(z)}{\varepsilon}
\end{array}\right] \\
A_{3}=\left(1-\frac{\tilde{K}_{e}(z)}{\tilde{K}^{a b s}}\right)\left(\frac{L}{T_{0} U} \partial_{t} w^{(2)}+v_{L O}^{(2)}+\int_{w^{(1)}}^{w^{(2)}} q d \xi\right)+\frac{\tilde{K}_{e}(z)}{\tilde{K}^{a b s}}\left(\frac{L}{T_{0} U} \partial_{t} w^{(1)}+v_{L O}^{(1)}\right), \\
A_{1}=A_{2}=0 .
\end{gathered}
$$

It remains to go back to the dimensional form. We set

$$
K^{H O M}(x, t)=-\frac{r-1}{r}\left(\left|x_{3}-\frac{h^{(1)}+h^{(2)}}{2}\right|^{r /(r-1)}-\left|\frac{h^{(1)}-h^{(2)}}{2}\right|^{r /(r-1)}\right),
$$

$$
\begin{gathered}
\text { For } x_{3} \geq\left(h^{(1)}+h^{(2)}\right) / 2, \quad K_{e}^{H O M}(x, t)=\left(h^{(2)}-h^{(1)}\right)^{r^{*}}\left(-2 x_{3}+h^{(2)}+h^{(1)}+\right. \\
\left.2 r^{*}\left(h^{(2)}-x_{3}\right)\right)+\left(2 x_{3}-h^{(2)}-h^{(1)}\right)^{1+r^{*}},
\end{gathered}
$$

$$
\begin{gathered}
\text { For } x_{3} \leq\left(h^{(1)}+h^{(2)}\right) / 2, \quad K_{e}^{H O M}(x, t)=r^{*}\left(h^{(2)}-h^{(1)}\right)^{1+r^{*}}+\left(h^{(1)}+h^{(2)}-\right. \\
\left.2 x_{3}\right)\left(\left(1+r^{*}\right)\left(h^{(2)}-h^{(1)}\right)^{r^{*}}-\left(h^{(1)}+h^{(2)}-2 x_{3}\right)^{r^{*}}\right), \\
K_{H O M}^{a b s}=\frac{\left(h^{(2)}-h^{(1)}\right)^{1+r^{*}}}{\left(1+r^{*}\right) 2^{r^{*}}} .
\end{gathered}
$$

Then 92 becomes

$$
\begin{gathered}
{\left[\begin{array}{c}
v_{1}^{F} \\
v_{2}^{F} \\
v_{3}^{F}
\end{array}\right]=\frac{K_{F}}{\eta_{F}^{r^{*}-1} \mid \mathbf{f}-\nabla p^{F} r^{r^{*}-2}\left(\mathbf{f}-\nabla p^{F}\right)}} \\
+\left[\begin{array}{c}
0 \\
0 \\
\left(1-\frac{K_{e}\left(x_{3}\right)}{K^{a b s}}\right)\left(\partial_{t} h^{(2)}+v_{\text {out }}^{(2)}+\int_{h^{(1)}}^{h^{(2)}} q d \xi\right)+\frac{K_{e}\left(x_{3}\right)}{K^{a b s}}\left(\partial_{t} h^{(1)}+v_{\text {out }}^{(1)}\right)
\end{array}\right],
\end{gathered}
$$

where the fracture absolute permeability is

$$
K_{F}=\left[\begin{array}{ccc}
K^{H O M} & 0 & -K_{e}^{H O M} \partial_{x_{1}} \log \frac{K_{H O M}^{a b s}}{K_{e}^{H O M}} \\
0 & K^{H O M} & -K_{e}^{H O M} \partial_{x_{2}} \log \frac{K^{a b s}}{K_{e}^{H O M}} \\
-K_{e}^{H O M} \partial_{x_{1}} \log \frac{K_{H b M}^{\text {abs }}}{K_{e}^{H O M}} & -K_{e}^{H O M} \partial_{x_{2}} \log \frac{K_{H M M}^{a b s}}{K_{e}^{H O M}} & K^{H O M}
\end{array}\right] .
$$


Furthermore,

$$
v_{L}=\left(1-\frac{K_{e}\left(x_{3}\right)}{K^{\text {abs }}}\right)\left(\partial_{t} h^{(2)}+v_{\text {out }}^{(2)}+\int_{h^{(1)}}^{h^{(2)}} q d \xi\right)+\frac{K_{e}\left(x_{3}\right)}{K^{\text {abs }}}\left(\partial_{t} h^{(1)}+v_{\text {out }}^{(1)}\right),
$$

where $v_{\text {out }}^{(j)}=-D h^{(j)} / D t$.

In the tip zone the permeability degenerates and Darcy's law 100 should not be used. For an advanced discussion of the interface conditions we refer to [49]. Further discussions on the singularity of the tip region can be found in $[43,25,62,35,26]$ and the many references cited therein.

\section{References}

[1] J. Adachi, E. Siebrits, A. Peirce, and J. Desroches. Computer simulation of hydraulic fractures. International Journal of Rock Mechanics and Mining Sciences, 44(5):739 - 757, 2007. ISSN 1365-1609. doi: http://dx.doi.org/10.1016/j.ijrmms.2006.11.006. URL http://www . sciencedirect.com/science/article/pii/S1365160906001870

[2] G. Allaire, F. Jouve, and N. V. Goethem. Damage and fracture evolution in brittle materials by shape optimization methods. Journal of Computational Physics, 230(12):5010 - 5044, 2011. ISSN 0021-9991. doi: http://dx.doi.org/10.1016/j.jcp.2011.03.024.

[3] M. Ambati, T. Gerasimov, and L. De Lorenzis. Phase-field modeling of ductile fracture. Computational Mechanics, pages 1-24, 2015. ISSN 0178-7675. doi: 10.1007/s00466-015-1151-4. URL http://dx.doi.org/10.1007/s00466-015-1151-4

[4] L. Ambrosio and V. Tortorelli. Approximation of functionals depending on jumps by elliptic functionals via $\gamma$-convergence. Comm. Pure Appl. Math., 43:999-1036, 1990.

[5] L. Ambrosio and V. Tortorelli. On the approximation of free discontinuity problems. Boll. Un. Mat. Ital. B, 6:105-123, 1992.

[6] H. Amor, J.-J. Marigo, and C. Maurini. Regularized formulation of the variational brittle fracture with unilateral contact: Numerical experiments. Journal of Mechanics Physics of Solids, 57:1209-1229, Aug. 2009. doi: 10.1016/j.jmps.2009.04.011.

[7] W. Bangerth, T. Heister, G. Kanschat, and M. others. Differential Equations Analysis Library, 2012.

[8] R. D. Barree and M. W. Conway. Experimental and numerical modeling of convective proppant transport. SPE-28564-MS, 1994. doi: doi:10.2118/28564-MS

[9] G. Beavers and D. Joseph. Boundary conditions at a naturally permeable wall. J. Fluid Mech., 30:197-207, 1967.

[10] R. Bird, R. Armstrong, R.C., and O. Hassager. Dynamics of polymeric liquids, volume Vol. 1 fluid mechanics. John Wiley and Sons, New York, 1987.

[11] M. J. Borden, C. V. Verhoosel, M. A. Scott, T. J. R. Hughes, and C. M. Landis. A phase-field description of dynamic brittle fracture. Comput. Meth. Appl. Mech. Engrg., 217:77-95, 2012.

[12] M. J. Borden, T. J. Hughes, C. M. Landis, and C. V. Verhoosel. A higher-order phase-field model for brittle fracture: Formulation and analysis within the isogeometric analysis framework. Comput. Methods Appl. Mech. Engrg., 273:100-118, 2014

[13] B. Bourdin. Numerical implementation of the variational formulation for quasi-static brittle fracture. Interfaces and free boundaries, 9: 411-430, 2007

[14] B. Bourdin, G. Francfort, and J.-J. Marigo. Numerical experiments in revisited brittle fracture. J. Mech. Phys. Solids, 48(4):797-826, 2000.

[15] B. Bourdin, G. Francfort, and J.-J. Marigo. The variational approach to fracture. J. Elasticity, 91(1-3):1-148, 2008.

[16] S. Burke, C. Ortner, and E. Süli. An adaptive finite element approximation of a variational model of brittle fracture. SIAM J. Numer. Anal., 48(3):980-1012, 2010.

[17] C. Burstedde, L. C. Wilcox, and O. Ghattas. P4est: Scalable algorithms for parallel adaptive mesh refinement on forests of octrees. SIAM J. Sci. Comput., 33(3):1103-1133, May 2011. ISSN 1064-8275. doi: 10.1137/100791634.

[18] R. H. Christopher and S. Middleman. Power-law flow through a packed tube. Ind. Eng. Chem. Fundamen., 4(4):422-426, 1965.

[19] P. E. Clark and J. A. Quadir. Prop transport in hydraulic fractures: A critical review of particle settling velocity equations. SPE-9866-MS, 1981. doi: doi:10.2118/9866-MS

[20] P. E. Clark, F. S. Manning, J. A. Quadir, and N. Guler. Prop transport in vertical fractures. SPE-10261-MS, 1981. doi: doi:10.2118/10261-MS.

[21] R. J. Clifton and J.-J. Wang. Multiple fluids, proppant transport, and thermal effects in three-dimensional simulation of hydraulic fracturing. SPE-18198-MS, 1988. doi: doi:10.2118/18198-MS.

[22] A. A. Daneshy. Numerical solution of sand transport in hydraulic fracturing. SPE-5636-MS, 1978. doi: doi:10.2118/5636-MS.

[23] C. Dawson, S. Sun, and M. Wheeler. Compatible algorithms for coupled flow and transport. Comput. Methods Appl. Mech. Engrg., 193: 2565-2580, 2004.

[24] R. Dean and J. Schmidt. Hydraulic-fracture predictions with a fully coupled geomechanical reservoir simulator. SPE Journal, 14(4):707-714, 2009.

[25] J. Desroches, E. Detournay, B. Lenoach, P. Papanastasiou, J. Pearson, M. Thiercelin, and A. Cheng. The crack tip region in hydraulic fracturing. Proc. R. Soc. Lond., 67:447, 1994.

[26] E. Detournay and A. Peirce. On the moving boundary conditions for a hydraulic fracture. Preprint - online, 2014.

[27] J. Donea, S. Giuliani, and J. Halleux. An arbitrary lagrangian-eulerian finite element method for transient dynamic fluid-structure interactions. Comput. Methods Appl. Mech. Engrg., 33:689-723, 1982.

[28] E. Dontsov and A. Peirce. Slurry flow, gravitational settling and a proppant transport model for hydraulic fractures. J. Fluid Mech., 760: 567-590, 2014.

[29] E. Dontsov and A. Peirce. Proppant transport in hydraulic fracturing: Crack tip screen-out in kgd and p3d models. International Journal of Solids and Structures, 63:206 - 218, 2015. ISSN 0020-7683. doi: http://dx.doi.org/10.1016/j.ijsolstr.2015.02.051. URL http://www . sciencedirect.com/science/article/pii/S0020768315001055

[30] D. Drew and S. Passman. Theory of multicomponent fluids. Applied Mathematical Sciences. Springer, 1999. 
[31] EPA. EPA final report: Evaluation of impacts to underground sources of drinking water by hydraulic fracturing of coalbed methane reservoirs. EPA number 816-R04-003, June 2004

[32] L. Formaggia and F. Nobile. A stability analysis for the arbitrary Lagrangian Eulerian formulation with finite elements. East-West Journal of Numerical Mathematics, 7:105 - 132, 1999.

[33] G. Francfort and J.-J. Marigo. Revisiting brittle fracture as an energy minimization problem. J. Mech. Phys. Solids, 46(8):1319-1342, 1998.

[34] B. Ganis, V. Girault, M. Mear, G. Singh, and M. Wheeler. Modeling fractures in a poro-elastic medium. Oil E Gas Science and TechnologyRevue dIFP Energies nouvelles, 69(4):515-528, 2014.

[35] D. Garagash and E. Detournay. The tip region of a fluid-driven fracture in an elastic medium. ASME J. Appl. Mech., 67:183-192, 2000.

[36] T. Gerasimov and L. D. Lorenzis. A line search assisted monolithic approach for phase-field computing of brittle fracture. Computer Methods in Applied Mechanics and Engineering, pages -, 2015. doi: http://dx.doi.org/10.1016/j.cma.2015.12.017. URL http://www . sciencedirect.com/science/article/pii/S0045782515004235

[37] G. Govier and K. Aziz. The flow of complex mixtures in pipes. Van Nostrand Reinhold Co., 1972. URL https://books.google.com/ books?id=bSRAAAAAIAAJ

[38] A. Griffith. The phenomena of rupture and flow in solids. Philos. Trans. R. Soc. Lond., 221:163-198, 1921.

[39] T. Heister, M. F. Wheeler, and T. Wick. A primal-dual active set method and predictor-corrector mesh adaptivity for computing fracture propagation using a phase-field approach. Comput. Methods Appl. Mech. Engrg., 290:466-495, 2015.

[40] M. Heroux, R. Bartlett, V. H. R. Hoekstra, J. Hu, T. Kolda, R. Lehoucq, K. Long, R. Pawlowski, E. Phipps, A. Salinger, H. Thornquist, R. Tuminaro, J. Willenbring, and A. Williams. An Overview of Trilinos. Technical Report SAND2003-2927, Sandia National Laboratories, 2003.

[41] T. Hughes, W. Liu, and T. Zimmermann. Lagrangian-Eulerian finite element formulation for incompressible viscous flows. Comput. Methods Appl. Mech. Engrg., 29:329-349, 1981.

[42] T. J. Hughes, G. Engel, L. Mazzei, and M. G. Larson. The continuous galerkin method is locally conservative. Journal of Computational Physics, 163(2):467 - 488, 2000. ISSN 0021-9991. doi: http://dx.doi.org/10.1006/jcph.2000.6577. URL http://www.sciencedirect. com/science/article/pii/S002199910096577X

[43] Y. Kovalyshen. Fluid-driven fracture in poroelastic medium. PhD thesis, The University of Minnesota, 2010.

[44] O. Ladyzhenskaja, V. Solonnikov, and N. Uralceva. Linear and quasi-linear equations of parabolic type. Translations of mathematical monographs, AMS Vol. 23, 1968

[45] B. Lecampion and E. Detournay. An implicit algorithm for the propagation of hydraulic fracture with a fluid lag. Comput. Meth. Appl. Mech. Engrg., 196:4863-4880, 2007.

[46] S. Lee, M. Wheeler, and T. Wick. Pressure and fluid-driven fracture propagation in porous media using an adaptive finite element phase field model. submitted, July 2015.

[47] S. Lee, Y.-J. Lee, and M. F. Wheeler. A locally conservative enriched galerkin approximation and user-friendly efficient solver for elliptic and parabolic problems. ICES report 15-19, submitted, September 2015.

[48] S. Malhotra, E. Lehman, and M. Sharma. Proppant placement using alternate-slug fracturing. SPE 163851, 2013.

[49] A. Marciniak-Czochra and A. Mikelić. Effective pressure interface law for transport phenomena between an unconfined fluid and a porous medium using homogenization. SIAM: Multiscale modeling and simulation, 10:285-305, 2012.

[50] M. McClure and R. Horne. Discrete fracture network modeling of hydraulic stimulation. Journal of applied physics, 2013.

[51] A. Mesgarnejad, B. Bourdin, and M. Khonsari. Validation simulations for the variational approach to fracture. Comput. Methods Appl. Mech. Engrg., 2014. online-available http://dx.doi.org/10.1016/j.cma.2014.10.052.

[52] C. Miehe and S. Mauthe. Phase field modeling of fracture in multi-physics problems. part iii. crack driving forces in hydro-poro-elasticity and hydraulic fracturing of fluid-saturated porous media. Computer Methods in Applied Mechanics and Engineering, pages -, 2015. ISSN 0045-7825. doi: http://dx.doi.org/10.1016/j.cma.2015.09.021. URLhttp://www.sciencedirect.com/science/article/pii/ S0045782515003151

[53] C. Miehe, M. Hofacker, and F. Welschinger. A phase field model for rate-independent crack propagation: Robust algorithmic implementation based on operator splits. Comput. Meth. Appl. Mech. Engrg., 199:2765-2778, 2010.

[54] C. Miehe, F. Welschinger, and M. Hofacker. Thermodynamically consistent phase-field models of fracture: variational principles and multifield fe implementations. International Journal of Numerical Methods in Engineering, 83:1273-1311, 2010.

[55] C. Miehe, M. Hofacker, L.-M. Schaenzel, and F. Aldakheel. Phase field modeling of fracture in multi-physics problems. part ii. coupled brittleto-ductile failure criteria and crack propagation in thermo-elasticplastic solids. Computer Methods in Applied Mechanics and Engineering, 294:486 - 522, 2015. ISSN 0045-7825. doi: http://dx.doi.org/10.1016/j.cma.2014.11.017. URL http://www.sciencedirect.com/ science/article/pii/S0045782514004435

[56] C. Miehe, L.-M. Schaenzel, and H. Ulmer. Phase field modeling of fracture in multi-physics problems. part i. balance of crack surface and failure criteria for brittle crack propagation in thermo-elastic solids. Computer Methods in Applied Mechanics and Engineering, 294:449 - 485, 2015. ISSN 0045-7825. doi: http://dx.doi.org/10.1016/j.cma.2014.11.016. URL http://www.sciencedirect.com/science/ article/pii/S0045782514004423

[57] A. Mikelić and R.Tapiéro. Mathematical derivation of the power law describing polymer flow through a thin slab. Modélisation Mathématique et Analyse Numérique (M⿻上丨), 29:3-22, 1995.

[58] A. Mikelić and M. F. Wheeler. Convergence of iterative coupling for coupled flow and geomechanics. Comput Geosci, 17(3):455-462, 2012.

[59] A. Mikelić, M. Wheeler, and T. Wick. Phase-field modeling of a fluid-driven fracture in a poroelastic medium. Computational Geosciences, 19 (6):1171-1195, 2015. ISSN 1573-1499. doi: 10.1007/s10596-015-9532-5. URL http://dx.doi.org/10.1007/s10596-015-9532-5

[60] A. Mikelić, M. F. Wheeler, and T. Wick. A phase-field method for propagating fluid-filled fractures coupled to a surrounding porous medium. SIAM Multiscale Model. Simul., 13(1):367-398, 2015.

[61] A. Mikelić, M. F. Wheeler, and T. Wick. A quasi-static phase-field approach to pressurized fractures. Nonlinearity, 28(5):1371-1399, 2015

[62] S. Mitchell, R. Kuske, and A. Peirce. An asymptotic framework for finite hydraulic fractures including leak-off. SIAM J. Appl. Math., 67(2): 364-386, 2007. 
[63] E. Novotny. Proppant transport. SPE-6813-MS, 1977. doi: http://dx.doi.org/10.2118/6813-MS.

[64] S. Ouyang, G. Carey, and C. Yew. An adaptive finite element scheme for hydraulic fracturing with proppant transport. Journal for Numerical methods in fluids, 24(7):645-670, 1997.

[65] A. Schlüter, A. Willenbücher, C. Kuhn, and R. Müller. Phase field approximation of dynamic brittle fracture. Comput. Mech., 54(5): 1141-1161, 2014. ISSN 0178-7675.

[66] B. A. Schrefler, S. Secchi, and L. Simoni. On adaptive refinement techniques in multi-field problems including cohesive fracture. Comput. Meth. Appl. Mech. Engrg., 195:444-461, 2006.

[67] A. Settari and D. A. Walters. Advances in coupled geomechanical and reservoir modeling with applications to reservoir compaction. SPE Journal, 6(3):334-342, Sept. 2001.

[68] E.-M. B. Shokir and A.A.Al-Quraishi. Experimental and numerical investigation of proppant placement in hydraulic fractures. SPE 107927MS, 2007.

[69] M. Smith, A. Bale, L. Britt, B. Halney, and H. Klein. Enhanced 2d proppant-transport simulation: The key to understanding proppant flowback and post-frac productivity. SPE 69211, 2001.

[70] I. N. Sneddon and M. Lowengrub. Crack problems in the classical theory of elasticity. SIAM series in Applied Mathematics. John Wiley and Sons, Philadelphia, 1969.

[71] S. Sun and J. Liu. A locally conservative finite element method based on piecewise constant enrichment of the continuous galerkin method. SIAM Journal on Scientific Computing, 31(4):2528-2548, 2009. doi: 10.1137/080722953.

[72] S. Sun and M. F. Wheeler. Projections of velocity data for the compatibility with transport. Computer Methods in Applied Mechanics and Engineering, 195(78):653 - 673, 2006. ISSN 0045-7825. doi: http://dx.doi.org/10.1016/j.cma.2005.02.011. URL http://www . sciencedirect.com/science/article/pii/S0045782505001234

[73] A. Z. Szeri. Fluid film lubrication, volume 2nd edition. Cambridge University Press, New York, 2011.

[74] I. Tolstoy. Acoustics, elasticity, and thermodynamics of porous media. Twenty-one papers by M.A. Biot. Acoustical Society of America, New York, 1992.

[75] L. A. Vadimovich. Models for proppant transport and deposition in hydraulic fracture simulation: A review of the state of the art. SINTEF rapport; 2011.057.

[76] C. V. Verhoosel and R. de Borst. A phase-field model for cohesive fracture. International Journal for Numerical Methods in Engineering, 96 (1):43-62, 2013. ISSN 1097-0207. doi: 10.1002/nme.4553. URL http://dx.doi.org/10.1002/nme.4553

[77] M. Wheeler, T. Wick, and W. Wollner. An augmented-Lagangrian method for the phase-field approach for pressurized fractures. Comp. Meth. Appl. Mech. Engrg., 271:69-85, 2014.

[78] T. Wick. Goal functional evaluations for phase-field fracture using pum-based dwr mesh adaptivity. RICAM Preprint No. 2015-47 at http://www.ricam.oeaw.ac.at/publications/reports/, 2015.

[79] T. Wick, G. Singh, and M. Wheeler. Fluid-filled fracture propagation using a phase-field approach and coupling to a reservoir simulator. accepted for publication in SPE Journal; ICES report 14-20, doi: 10.2118/168597-PA, Aug 2014.

[80] T. Wick, S. Lee, and M. Wheeler. 3d phase-field for pressurized fracture propagation in heterogeneous media. VI International Conference on Computational Methods for Coupled Problems in Science and Engineering 2015 Proceedings, May 2015. 